\title{
Mechanisms of allergen-specific B cell tolerance in children with cow's milk-oral immunotherapy and natural outgrowth of milk allergy
}

\section{Pattraporn Satitsuksanoa}

Swiss Institute of Allergy and Asthma Research (SIAF), University of Zürich https://orcid.org/00000001-9540-7759

\section{Willem van de Veen}

Swiss Institute of Allergy and Asthma Research (SIAF), University of Zü https://orcid.org/0000-00019951-6688

\section{Ge Tan}

Functional Genomics Center Zurich https://orcid.org/0000-0003-0026-8739

\section{Oliver Wirz}

Sean N. Parker Center for Allergy and Asthma Research

\section{Kirstin Jansen}

Swiss Institute of Asthma and Allergy Research https://orcid.org/0000-0002-9176-8562

\section{Milena Sokolowska}

Swiss Institute of Allergy and Asthma Research (SIAF), University of Zurich https://orcid.org/00000001-9710-6685

\section{David Mirer}

Swiss Institute of Allergy and Asthma Research (SIAF), University of Zürich

\section{Anna Globinska}

Swiss Institute of Allergy and Asthma Research (SIAF), University of Zürich

\section{Tadech Boonpiyathad}

Swiss Institute of Allergy and Asthma Research (SIAF), University of Zürich

\section{Stephan Schneider}

Swiss Institute of Allergy and Asthma Research (SIAF), University of Zürich

\section{Elena Barletta}

Swiss Institute of Allergy and Asthma Research (SIAF), University of Zürich https://orcid.org/00000002-8431-4140

\section{Hergen Spits}

The department of Experimental Immunology of the Amsterdam University Medical Centers Iris Chang

Stanford University

\section{Scott Boyd}


Stanford University https://orcid.org/0000-0003-0963-044X

\section{Cezmi Akdis}

Swiss Institute of Allergy and Asthma Research (SIAF) https://orcid.org/0000-0001-8020-019X

\section{Kari C. Nadeau}

Stanford University https://orcid.org/0000-0002-2146-2955

\section{Mübeccel Akdis ( $\square$ akdism@siaf.uzh.ch )}

Swiss Institute of Allergy and Asthma Research

\section{Article}

Keywords: Oral allergen-specific immunotherapy (OIT), allergen-specific B cells, alphaS1-casein (aS1casein), cow's milk allergy, natural tolerance, RNA-sequencing

Posted Date: December 16th, 2021

DOI: https://doi.org/10.21203/rs.3.rs-1134709/v1

License: (c) (1) This work is licensed under a Creative Commons Attribution 4.0 International License. Read Full License 


\section{Abstract}

Antigen-specific memory B cells play a key role in the induction of immune tolerance to food allergens and clinical healing. Here, we characterized the role of allergen-specific B cells in immune tolerance induced by oral allergen-specific immunotherapy (OIT) and natural tolerance that developed in children who spontaneously outgrew cow's milk allergy. Increased frequency of circulating milk allergen $\mathrm{aS}_{1^{-}}{ }^{-}$ casein -specific B cells was observed after OIT and natural tolerance (NT). Milk desensitized subjects showed partial acquisition of tolerance phenotypic features induced tolerance, suggesting that desensitization is an earlier stage of tolerance. Immunoregulatory genes such as IL 1ORA and IGHG4 are significantly upregulated after OIT (desensitized and tolerance) versus NT. Secreted proteins from allergen-specific B cells revealed higher amounts of regulatory cytokines, IL-10 and TGF- $\beta$ after OIT and NT. Taken together, allergen-specific B cells are essential elements in regulating food allergen tolerance in both OIT-received and naturally-resolved individuals.

\section{Introduction}

The prevalence of food allergy has been increasing in recent decades and affects approximately $10 \%$ of the world population ${ }^{1,2}$. Cow's milk allergy (CMA) is a common disease in infants and children that shows a high rate of spontaneous resolution in early childhood until adolescence ${ }^{3}$. In some cases, CMA can result in anaphylactic reactions and has long-term implications for growth and nutrition. Avoidance of all cow's milk products in daily life is a standard physicians' recommendation to patients ${ }^{4}$. As an alternative to eliminating cow's milk from the patient's diet, oral allergen-specific immunotherapy (OIT) is an effective and curative treatment inducing clinical and immunologic tolerance to milk allergens in allergic patients ${ }^{5,6}$. Understanding immune tolerance mechanisms to food allergens is crucial for further improving the existing treatments, and for the discovery of novel ways to prevent and treat food allergies $^{7,8}$.

There are two major mechanisms which classify allergic reactions to cow's milk and other food allergens; immunoglobulin E (IgE)-mediated and non-IgE-mediated ${ }^{9,}{ }^{10}$. The development of IgE-mediated CMA is regulated by $B$ cells through the production of allergen-specific lgE antibodies ${ }^{11}$. Mechanisms driving $B$ cells responses during allergy and development to tolerance remain to be elucidated. Hence, the investigation of B cell responses in food-allergic patients during OIT and in individuals who outgrow food allergy due to natural immune tolerance development may clarify mechanisms of induction and maintenance of food allergen tolerance.

Natural outgrowth of food allergies represents a valuable model to study mechanisms of immune tolerance to food allergens. So far, there have been very few well-designed studies regarding the natural development of tolerance to food allergens. Reported rates of resolution (natural outgrowth) vary widely, likely attributable to methodological differences and study populations. Some food allergens are difficult to avoid and fortunately have a generally high likelihood of natural outgrowth, such as cow's milk and 
egg $^{2,12,13}$. Safely loosening the diet to include milk and egg for children has important nutritional and quality-of-life benefits. It was demonstrated that tolerant children, who outgrew their allergy developed higher frequencies of circulating CD4+CD25+ Treg cells and decreased in vitro proliferative responses to bovine beta-lactoglobulin compared to children who maintained clinically active allergy ${ }^{13}$. Other food allergen sources, particularly peanuts and tree nuts, have a much lower spontaneous resolution rate and therefore require an efficient OIT approach ${ }^{2,12}$.

Molecular mechanisms of spontaneous outgrowth of food allergies have not been studied in detail and there is no report on the role of allergen-specific B cells in natural tolerance. Hoh et al. investigated B cell responses in peanut allergy and demonstrated that local class-switch recombination (CSR) to IgE in the gut directly has a major impact on the development of food allergen-specific IgE antibodies ${ }^{14}$. Circulating $\lg \mathrm{E}+\mathrm{B}$ cells display mostly immature plasmablast phenotype ${ }^{15}$. Increased numbers of circulating lgE+ memory B cells and IgE+ plasmablasts are correlated with the presence of food allergy and may contribute to pathogenesis ${ }^{16}$.

Here, we have performed an in-depth characterization of $B$ cells specific for $\mathrm{aS}_{1}$-casein, the major allergen in cow's milk, to analyse B cell changes related to the development of tolerance to cow's milk allergy. We identify an increased frequency of allergen-specific B cells in OIT induced-cow's milk tolerance and natural outgrowth-related immune tolerant individuals. Allergen-specific B cells predominantly express IgG4 after OIT and natural tolerance, with smaller numbers expressing $\lg \mathrm{G} 1$ or $\lg \mathrm{E}$. Gene expression signatures associated with allergen-specific $B$ cells were mostly downregulated after OIT, showing that allergen-specific $B$ cells decrease their expression of 1456 genes and downregulate activation and proinflammatory gene expressions profile. Notably, secreted protein profiles of specific B cells were similar after OIT and NT, suggesting common but not identical mechanisms of food allergen tolerance.

\section{Results}

\section{Increased frequency of $\mathrm{aS}_{1}$-casein-specific $B$ cells in OIT- induced tolerance and natural tolerance}

The frequencies of $\mathrm{aS}_{1}$-casein-specific $\mathrm{B}$ cells (CD19+IgM-CD27+) in circulation was calculated by direct analyses of the cells without culturing or other ex vivo manipulations. The details of the gating strategy are shown in supplementary Fig. 1. $\mathrm{aS}_{1}$-casein-specific $\mathrm{B}$ cells were found at low frequencies of $0.01-$ $0.5 \%$ in three main representative groups including healthy control $(\mathrm{HC})$, allergic groups (before OIT, during OIT, and after OIT), and natural tolerance (NT) (Fig. 1A). Mean frequencies \pm standard deviation was $0.03 \pm 0.03$ in $\mathrm{HC}, 0.16 \pm 0.06$ before OIT, $0.29 \pm 0.13$ in during OIT, $0.28 \pm 0.14$ in after OIT, and $0.37 \pm 0.15$ in NT (Fig. 1B). A significant increase in allergen-specific B cell frequency was observed in the course of OIT. The highest frequency of allergen-specific B cell was in the NT group. While HC exhibited the lowest frequency of $\mathrm{aS}_{1}$-casein-specific $B$ cells. These data demonstrate that allergen-specific $B$ cell frequencies are increased during OIT and NT development. 


\section{aS ${ }_{1}$-casein-specific B cells produce predominantly IgG4 in OIT and natural tolerance}

To verify allergen-specificity and isotype expression of $\mathrm{aS}_{1}$-casein-specific $B$ cells, we detected specific Abs including slgG, slgG1, slgG2, slgG3, slgG4, slgA1, slgA2, and slgE in supernatants from $5 \times 10^{5}$ immortalized $\mathrm{aS}_{1}$-casein-specific and non-specific $B$ cells with $0.6 \mu \mathrm{M} \mathrm{aS}_{1}$-casein allergen stimulation for 72 hours.

From the ELISA results, $\mathrm{aS}_{1}$-casein-specific IgG was $0.39 \pm 0.16$ in $\mathrm{HC}+, 0.5 \pm 0.08$ in before OIT,$+ 0.64 \pm 0.11$ in after $\mathrm{OIT}+$, and $0.84 \pm 0.07$ in $\mathrm{NT}+\mathrm{aS}_{1}$-casein-specific $\operatorname{lgG} 1$ was $0.13 \pm 0.02$ in $\mathrm{HC}+, 0.24 \pm 0.07$ in before

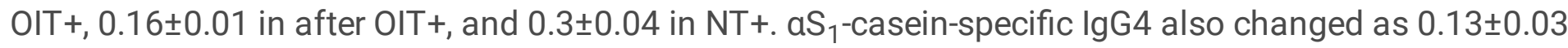
in $\mathrm{HC}+, 0.17 \pm 0.05$ in before OIT,$+ 0.41 \pm 0.06$ in after OIT+, and $0.53 \pm 0.09$ in NT+. aS ${ }_{1}$-casein-specific IgE was $0.03 \pm 0.01$ in $\mathrm{HC}+, 0.26 \pm 0.05$ in before OIT+, $0.22 \pm 0.07$ in after OIT+, and $0.12 \pm 0.02$ in NT+. In contrast, slgG2, slgG3, slgA1, and slgA2 were undetectable or very low in B cell supernatants (data not shown). The production of $\mathrm{aS}_{1}$-casein-slgG, slgG1, slgG4, and slgE was significantly higher in specific $B$ cells from HC, before OIT, after OIT, and in NT compared to the non-specific B cells (Fig. 2A). These results confirm that the sorted single allergen-specific $\mathrm{B}$ cell populations were highly pure and expanded. Importantly, we observed that $\mathrm{aS}_{1}$-casein-specific $\mathrm{B}$ cells from healthy individuals did not produce slgE and rather showed slgG1 and slgG4 production. In contrast, food-allergic individuals had relatively high slgE production together with slgG1 and relatively low slgG4 isotype Abs. The levels of these Abs showed a very significant change after OIT. slgG4 became the major Ab isotype with quite low slgG1 without showing any change in slgE Abs. For comparison, NT demonstrated a high slgG4 Ab response with moderately low slgG1 and slgE. To further demonstrate this change in the prevailing specific Ab isotype, the slgE/slgG4 ratio was calculated in all groups. In the NT and HC $(0.24 \pm 0.05$ and $0.48 \pm 0.56$, respectively), the slgE/slgG4 ratios were significantly lower compared to before OIT $(1.65 \pm 0.7)$ and after OIT ( $0.55 \pm 0.2$, respectively). Allergic patients before OIT had the highest IgE/lgG4 ratio, whereas the natural tolerance group had the lowest. (Fig. 2B).

\section{Downregulation of aS ${ }_{1}$-casein-specific $B$ cells in OIT-induced desensitization and significant silencing in tolerance}

To further delve into the in vivo functional changes in $\mathrm{aS}_{1}$-casein-specific $\mathrm{B}$ cells that could be related to immune tolerance, they were freshly isolated and immediately analysed using the ultra-low input RNAsequencing method without any cell culturing. Differentially expressed genes (DEGs) were identified in the gene expression profile of $\mathrm{aS}_{1}$-casein-specific and non-specific $B$ cells from before and after OIT with 2 outcomes: desensitized and tolerance (Fig. 3A). OIT outcomes were defined based on allergic reaction results from cow's milk extract re-challenge at six weeks after the immunotherapy. Patients who showed any reaction more than $32.7 \mathrm{mg}$ dose during the challenge, were classified as desensitized. Patients who tolerated the 8,000-12,000 mg dose without any clinical reactions were identified as tolerance patients. Immunotherapy-induced genes were defined as DEGs in comparisons of pre-OIT and after OIT where the $p$-value was lower than 0.05. RNA-seq data from participants with desensitized and tolerance outcomes 
after OIT (4 desensitized and 5 tolerance) were analysed. Fig. 3A shows the top 200 most significantly downregulated genes in $\mathrm{aS}_{1}$-casein-specific $\mathrm{B}$ cells after OIT compared to before OIT. There was a clear difference in gene expressions between desensitized and tolerance groups. The desensitized group showed down-regulation of certain clusters of genes compared to the pre-OIT state, but the tolerance group showed a markedly greater decrease in expression of these genes, approaching a complete lack of expression in some cases. In contrast, there was no change in expression of these genes pre- and postOIT in non-specific B cells representing the switched memory cells. Biological pathways potentially affected by DEGs were further analysed using the Metacore and Enrichr programs. Ten B cell-related pathways including immune response-mediated by circulating Igs, regulation of B cell activation, B cell receptor signalling, cytokine-mediated signalling, antigen receptor-mediated signalling, cellular response to cytokine stimulus, inflammatory response, regulation of immune response, $B$ cell activation involved in immune response, and chemokine-mediated signalling were identified (Fig. 3B, supplementary table 1). A volcano plot from aS ${ }_{1}$-casein-specific $B$ cells after OIT versus before OIT showed 1,456 significantly downregulated genes and 40 significantly upregulated genes (Fig. 3C).

To characterize the genes that were upregulated after OIT by their roles and functions from the literatures search, we compared B cell-related DEGs between desensitized and pre-OIT samples in Fig. 3D. B cell antigen-binding genes (TAP1, ITGA4, EP300) were significantly increased in the desensitized patients compared to before OIT. B cell cytokines and their receptors; IL 12RB2 and TNFRSF10D were completely downregulated in the desensitized group. B cell activation showed higher expression of TLR4 in the allergic group that decreased during desensitization, but FOXP1 and JAK3 were increased in the desensitized group. B cell chemokine genes including PLAU, CX3CR1, CCL3L3, XCL2 showed a significant decrease in the desensitized group. In contrast, CCR6, a gut homing chemokine receptor was highly expressed in desensitized patients. Breg cell-related genes including $A H R, G Z M B, G Z M H$ decreased during desensitization, but IL1ORA and TGFB3 showed a very significant increase. BCR signalling (PIK3CA, PIK3CD, PIK3CG), B cell differentiation (BCL11A, CARD11, DOCK11, MALT1), as well as B cell immunoglobulin genes (IGHG2) showed a significant increase after desensitization.

In the comparisons between the allergic and tolerance groups (Fig. 3E), we found that B cell cytokines/cytokine receptors (IL 1RAP, IL 12RB2, IL 18R1) and B cell chemotaxis (PLXNA1, PLXNA2, XCL 1, $X C L 2$ ) showed a decrease in the tolerance group after OIT. Interestingly, IGHG4 which has been repeatedly reported in other allergen tolerance studies showed a significant increase in the tolerance group ${ }^{17}$. Other Breg cell-related genes (TGFB3, ACVR1C, ACVR2B) had low expression. In contrast, B cell immunoglobulins constant regions (IGHG1, IGHA1, IGHA2, IGHD) are highly increased in tolerance subjects. Notably, the IGHD gene encodes the heavy chain of IgD, which was previously reported to increase in serum from cow's milk-allergy resolved cohort ${ }^{18}$.

These data demonstrate clear differences in the gene expression signatures of desensitized, and tolerance patient's allergen-specific B cells compared to the pre-OIT state. A large number of B cell activation, cytokine, chemotaxis and differentiation-related genes are downregulated after OIT. Our 
findings suggest that specific B cells under the influence of OIT, downregulate the cellular activation gene machinery in desensitized patients and mostly shut down during the tolerance state.

\section{Differences in aS ${ }_{1}$-casein-specific B cells between OIT-induced desensitization and tolerance}

We further analysed the overall gene data sets with a principal component analysis (PCA). This PCA plot demonstrates the top 200 DEGs that are separated into 4 different groups; before OIT, desensitized, tolerance, and NT group (Fig. 4A). DEGs characteristic of each group and clinical outcome were identified. $B$ cells from patients with desensitization outcomes had intermediate results in comparison to pre-OIT and tolerance patient $B$ cells. We evaluated the distinctive OIT-induced genes in desensitized and tolerance groups. Fig. $4 \mathrm{~B}$ and $4 \mathrm{C}$ show that $\mathrm{B}$ cell antigen-binding (TAP1), $\mathrm{BCR}$ signalling (PIK3CA, $P I K 3 C D, P R K C B$ ), B cell activation (RIF1, RNF168, SWAP70, FOXP1, JAK3), B cell chemotaxis (CCR6), B cell cytokines/cytokine receptors (IL21R), B cell differentiation (BCL11A, CARD11, DOCK11, MALT1), and Breg cell-related genes (IL 1ORA) were further decreased in tolerance patients compared to desensitized patients. In contrast, TLR4 showed a significant increase after tolerance. Moreover, B cell immunoglobulins (IGHG1, IGHA1, IGHA2) were highly expressed in tolerance patients, suggesting B cell class switch recombination in relation to antigen-specificity and plasma cell differentiation in tolerance memory B cells.

\section{Distinct gene signatures of aS ${ }_{1}$-casein-specific $B$ cells between outgrowth from milk allergy by natural tolerance compared to milk allergic children}

This study also enabled us to delve into mechanisms of natural tolerance. Accordingly, we compared the differences in gene expression signatures between $\mathrm{aS}_{1}$-casein-specific $B$ cells from food allergic individuals versus NT in circulating antigen-specific B cells representing the in vivo condition. A heatmap of DEGs highlights differences in the gene expression profile of $\mathrm{aS}_{1}$-casein-specific $B$ cells before OIT compared to NT (Fig. 5A). B cell-related pathways were analysed and shown in Fig. 5B and supplementary table 2 . A volcano plot from aS ${ }_{1}$-casein-specific B cells before OIT versus NT show 1,693 significantly downregulated genes and 704 upregulated genes (Fig. 5C). Although regulation of gene expression suggested less B cell activation and induced allergen tolerance during OIT, we did not see the same type of changes in NT. These findings suggest that different types of mechanisms may play a role in NT. Interestingly, the changes in NT were relatively heterogeneous compared to the gene expression of specific B cells in allergic individuals. Allergic children showed a high number of significantly increased $B$ cell activation-related genes. Many of them were significantly downregulated in specific B cells upon NT (Fig. 5D). A significant number of antigen-specific B cell activation molecules was downregulated in NT. An exciting finding was the expression of type 2 cytokine receptor genes (IL4R, IL 13RA) was significantly lower in NT allergen-specific B cells compared to those of allergic children. Significantly lower expression of antigen-presentation-related costimulatory receptors (CD40,TAP1), B cell activation (BATF, HRH2, $T L R 2, A D A$ ), cytokines or cytokine receptors (ILTR, IL 12RB1, IL12RB2, IL18R1, IL23A), chemotaxis (PLAU, CXCR3, XCL1, XCL2, CCL3L3, CCL4L2, CCRL2), Breg-related genes (GZMB, GZMH, ACVR1B, TGFB3), BCR signalling genes (PIK3R3), and B cell differentiation (HMGB3) was also seen in NT. 
NT was associated with a group of upregulated genes in B cells, such as NOTCH2, FOXP1, TLR1, TLR6, $T L R 9$, cytokine/cytokine receptors (IL21R, IL2RG) that are important in clonal expansion of established tolerance related clones, and chemokine genes (TNFRSF1B), BCR signalling (CD22, PIK3CD), B cell differentiation (BCL6, CARD11) and immunoglobulin genes (IGHD). Several of these genes display a direct or indirect relationship to immune tolerance and plasma cell differentiation. These data indicate a complementary shutdown mechanism by allergen-specific B cells in natural tolerance and OIT-induced tolerance, however, regarding their immune-tolerance-related properties and kinetics, DEGs do not exactly span the same molecules in their roles and biological functions.

\section{Comparison of gene signatures of aS ${ }_{1}$-casein-specific B cells in OIT-induced tolerance and natural tolerance}

To compare gene signatures between $\mathrm{aS}_{1}$-casein-specific $B$ cells from tolerance-induced after OIT versus NT, we first generated a heatmap of most significant DEGs (Fig. 6A). B cell-related pathways were analysed and shown in Fig. 6B and supplementary table 3, which showed similar pathways in induced tolerance and NT. A volcano plot from aS ${ }_{1}$-casein-specific B cells after OIT versus NT showed 1,303 significantly downregulated and 552 upregulated genes (Fig. 6C). 358 genes showed similar changes in OIT and NT patient B cells compared to pre-OIT samples, indicating some similar mechanisms in tolerance-induction by OIT and NT (Fig. 6D).

We observed that B cell activation (STAT6, FOXP1, NOTCH2, TLR9), B cell cytokine/cytokine receptors (IL2RG, IL7), Breg cell-related genes (AHR), B cell differentiation (BCL6, CARD11, DOCK11), BCR signalling $(C D 22, P I K 3 C A)$, and immunoglobulin (IGHD) are high in NT group. In contrast, OIT induced tolerance displayed higher levels of gene expression in antigen-binding (CD40,TAP1), cytokine/cytokine receptors (IL23A, IL21R), Breg cell-related genes (IL 10RA), B cell differentiation (CD79A, CD79B), and immunoglobulin (IGHG1, IGHG4, IGHA1, IGHA2) (Fig. 6E).

Gene signatures from immunotherapy-induced tolerance in specific B cells show marked changes compared to the pre-OIT state. However, these changes did not fully overlap with those seen in patients who achieved natural tolerance. Most importantly, IL 10RA and IGHG4 genes encoding cytokine IL-10 and IgG4 were expressed more in the induced-tolerance state.

\section{Secreted proteins from aS ${ }_{1}$-casein-specific $B$ cells in children with cow's milk induced tolerance and natural tolerance display similarity}

We further analysed the secreted proteins from purified $\mathrm{aS}_{1}$-casein-specific $B$ cells, that were immortalised and expanded. Proximity extension assay (PEA) technology was used to measure 92 proteins in a multiplex assay (inflammation panel). Heatmap of differentially expressed proteins showed significantly secreted proteins from specific B cells before OIT, after OIT (tolerance), and NT (Fig. 7A). The GO Biological processes pathways were analysed and shown in Fig. 7B and supplementary table 4 . The 
23 most significant differentially expressed proteins are shown in Fig. 7C and the remaining of nonsignificant plots are included in supplementary Fig. 3.

As a major finding, we observed that the secretion of immunoregulatory cytokines IL-10 and latency activated protein (LAP) of TGF-beta (TGF- $\beta$ ), that belong to $B$ cell activation pathway, were increased in the supernatants of specific B cells from the NT group. Similarly, several proteins including adenosine deaminase (ADA), caspase-8 (CASP-8), IL-7, were also increased in NT compared to before OIT. From chemotaxis pathway; urokinase-type plasminogen activator (UPA), cytokine-mediated signalling pathway; macrophage colony-stimulating factor 1 (CSF-1), Fms-related tyrosine kinase 3 ligand (Flt3L), tumor necrosis factor receptor superfamily member 9 (TNFRSF9), and cell-surface receptor signalling pathway; 4E-binding protein 1 (4E-BP1), STAM-binding protein (STAMBP), metalloproteinase-1 (MMP-1), axin-1 (AXIN1), osteoprotegerin (OPG) were also increased.

Interestingly, 13 proteins in these four different pathways such as LAP TGF- $\beta$, TNFRSF9, AXIN1, uPA, STAMBP, C-X-C Motif Chemokine Ligand 10 (CXCL10), C-C Motif Chemokine Ligand 20 (CCL20), vascular endothelial growth factor A (VEGFA), and IL-6 were significantly increased in after OIT-induced tolerance when compared to before OIT. In contrast, monocyte chemotactic protein 1 (MCP-1), monocyte chemotactic protein 4 (MCP-4), matrix metalloproteinase-10 (MMP-10), and Artemin (ARTN) were highly expressed in OIT-induced tolerance group. In addition, 7 proteins, TNFRSF9, ADA, and CSF-1 showed higher expression in NT compared to OIT-induced tolerance, but AXIN1, MMP-10, ARTN, and IL-6 showed lower expression.

In conclusion, the majority of secreted protein molecules from allergen-specific B cell cultures that display immune-regulatory and anti-inflammatory properties are highly secreted in natural tolerance and inducedtolerance as compared to allergic conditions.

\section{Discussion}

The present study provides an in-depth investigation of allergen-specific B cells from children with cow's milk-induced tolerance and NT. Although cow's milk is one of the most important and basic nutrients introduced early in life in the diet, it can induce lgE-associated food allergy that causes severe allergic manifestations in the gut, skin, and even in the respiratory tract and may lead to life-threatening anaphylactic shock. The major cow's milk allergens belong to the casein group of proteins $\left(\mathrm{aS}_{1}, \mathrm{aS}_{2}-, \beta-\right.$, and k-casein) and whey proteins (a-lactalbumin and $\beta$-lactoglobulin) ${ }^{19,20}$. We focused our investigation on a direct in vivo analysis of freshly isolated $\mathrm{aS}_{1}$-casein allergen-specific $B$ cells not manipulated by cell

culture or other procedures. Additional experiments with these low-frequencies of specific B cells included immortalization of them to confirm their antigenic specificity, and measurement of cytokine expression profiles.

Our approach in the identification of $\mathrm{aS}_{1}$-casein-specific $B$ cells was performed with a double-allergen labelling method that was established in several studies including the identification of peanut-specific $B$ 
cells (Ara h1 and Ara h2) ${ }^{21}$, bee venom-specific B cells (PLA) ${ }^{22}$, and house dust mite-specific B cells (Der $p 1)^{23}$. We found significantly increased percentage of circulating allergen-specific $B$ cells in allergic individuals, which continued to increase during, and after OIT. Increased frequencies of allergen-specific B cells were demonstrated in a previous study in house dust mite allergic patients after 2 years of immunotherapy ${ }^{23}$. We observed very low frequencies of allergen-specific B cells in HC. Interestingly, the highest frequencies of allergen-specific $B$ cells were observed in NT, suggesting the in vivo expansion of functional allergen-specific B cells during spontaneous outgrowth of allergies probably due to continuous exposure afterward.

Indeed, we confirmed the specificity of sorted B cells for $\mathrm{aS}_{1}$-casein, and our results exhibited the higher levels of $\mathrm{aS}_{1}$-casein-slgG, slgG1, slgG4, and slgE in supernatants of specific B cells. In addition, we detected slgE in the milk allergic and OIT-receiving group, but not in $\mathrm{HC}$ and it was at the low levels in NT. slgG4 isotype was predominantly high after OIT and in the NT group. It has been demonstrated in other allergens, such as bee venom, egg, and house dust mites that exposure to allergens induces an immunologic response that specifically enhances $\lg G 4$ levels due to continuous allergen exposure $23,24,25$, 26. The allergic group showed a significant decrease in slgE/slgG4 ratios after OIT, and even lower slgE/slgG4 ratios were observed in the NT group and HC. Therefore, low slgE and slgG4 Ab levels in cow's milk allergic children may be a predictive marker in the achievement of natural tolerance and successful response to $\mathrm{OIT}^{27}$.

There was a clear difference between tolerance and desensitized B cells suggesting that desensitization is an intermediate step on the way to tolerance development. In both the tolerance and desensitized groups, antigen binding, cytokines and receptors, B cell activation, chemotaxis, BCR signalling, B cell differentiation, and $B$ cell Ig heavy chain genes were altered. There was an increase of Breg cell-related genes, BCR signalling and differentiation. Within these most significantly increased genes, IL 1ORA, a receptor for the binding of suppressor cytokine IL-10 and TGFB3 a regulatory cytokine for antigen/allergen tolerance were highly expressed in desensitized OIT patients. Besides, the expression of CCR6, a gut homing chemokine ${ }^{28}$, was also higher in desensitized individuals, suggesting the OITinduced-tolerance state might occur in the gut.

Changes in the expression of B cell IGH genes showed interesting findings. While in the desensitized patients, only an increase in IGHG2 was observed, in tolerance subjects, we saw a significant increase in genes encoding the heavy chains for $\lg G 4, \lg G 1, \lg A 1, \lg A 2$, and $\lg D$. An increase in all of these specific antibody isotypes has been linked to tolerance development in serum in AITs with different allergens ${ }^{27,29,}$ 30. Among all immunoglobulin isotypes, IgG4 always appeared in the forefront as an immune tolerancelinked antibody isotype particularly induced by $\mathrm{IL}-10^{31}$.

The mechanisms of NT development were investigated in two ways. First, it was compared with the allergic situation before OIT, and second, it was compared with tolerance induced after OIT. The main finding was a similar downregulation of many B cell genes in NT. A type 2 immune response (IL4R and 
IL 13RA7) is characteristic of allergic response as also observed in food allergy and a costimulatory receptor $(C D 40)$ of $\mathrm{B}$ cells were significantly lower in $\mathrm{NT}$, proposing the inhibition of allergic responses ${ }^{32}$. Similarly, TAP1 which plays a role in the antigen-presentation of B cells was lower in NT suggesting that the antigen-presentation capacity of specific $B$ cells was downregulated ${ }^{33}$. The other downregulated genes, such as $B A T F, H R H 2, T L R 2$, and $A D A$ that are associated with allergic diseases were all downregulated in $\mathrm{NT}^{34,35,36}$. $A D A$ is also associated with elevated $\operatorname{lgE}$ in atopic and food allergic patients $^{37,38}$. NT decreases several B cell cytokines or cytokine receptors such as, $I L 7 R$ that controls homeostasis of allergen-specific memory CD4+T cells in the lung and airways ${ }^{39}, I L 12 R B 1$ and IL 12RB2 that contribute to allergen-induced airway inflammation in asthma ${ }^{40} / L 18 R 1$ that is involved the pathophysiology of allergic diseases ${ }^{41}$, and $I L 23 A$ enhances Th2 polarization and regulates allergic airway inflammation ${ }^{42}$. As a part of specific $B$ cell suppression in NT, chemotaxis genes including PLAU known as Treg suppressor, CXCR3 that binds to chemokine CXCL-10 and enhances antigen-specific Th1 responses in healthy humans ${ }^{43}, X C L 1$ and $X C L 2$ that play a crucial role in intestinal immune homeostasis ${ }^{44}$, along with chemokine genes $C C L 3 L 3, C C L 4 L 2, C C R L 2$ that control airway inflammatory responses are all down regulated ${ }^{45}$. In addition, $G Z M B$ and $G Z M H$ that act as mediators of allergic inflammation in human asthma ${ }^{46}, T G F B 3$ and its family gene $A C V R 1 B$ that act in the initiation and effector phases of allergic disease, as well as in consequent tissue dysfunction ${ }^{47}$, and PIK3R3 gene that also signals in B cell co-stimulation were also down regulated ${ }^{48}$.

Some of the genes that contribute to the B cell activation were upregulated, such as NOTCH2 that suppresses food antigen-induced mucosal mast cell hyperplasia ${ }^{49}$ and mediates the development and plasticity in marginal zone B cell ${ }^{50,51}$. FOXP1 that plays a critical role in human plasma cell differentiation was upregulated ${ }^{52}$. Two cytokine receptors $I L 21 R$ and IL2RG via STAT3-dependent induction of transcription factors required for $B$ cell expansion and plasma cell generation were increased in specific cells in $\mathrm{NT}^{53}$. The TNFRSF1B, a member of TNFRSF family, which acts as a co-signalling chemokine in BAFFR system for the B cell activation was increased in $\mathrm{NT}^{54}$. Toll-like receptors genes expression (TLR1, TLR6, TLR9) on B cells in NT, displayed the potential to induce secretion of cytokines, chemokines, and regulate immune homeostasis ${ }^{55}$. In addition, TLR9 is an important stimulus for Breg cell development ${ }^{22}$. CD22 was increased in NT, is an essential B cell suppressor molecule ${ }^{56}$ which plays a role in allergen tolerance ${ }^{57}$. PIK3CD was also increased in NT, suggesting a role in the control of $B$ cell development and function ${ }^{58}$. The significantly increased gene in specific B cells, BCL 6 suppresses IL-4 production in memory phenotype Th2 cells and suppresses Th2 immune responses in allergies ${ }^{59}$.

CARD11 mutations affect $\mathrm{B}$ cell development ${ }^{60}$ and cause cellular defects in congenital lymphoproliferative patients and atopic dermatitis individuals ${ }^{61,62}$. In CMA and house dust mite allergic patients, allergen-specific IgD was secreted after the course of OIT in serum samples ${ }^{18,63}$.

In the present study, we compared two types of immune tolerance to allergens, namely the natural and OIT-induced tolerance in specific B cells. One of the main differences between OIT-induced tolerance and 
NT is that NT is a long-lasting tolerance, whereas OIT-induced tolerance has been recently established, and there is no guarantee that it will persist for long periods. It has been shown that viral infections can revert this kind of tolerance even in the course of OIT $^{64}$. As a matter of fact, when we looked at the DEGs we can see that a significant number of genes show similar changes in expression in both OIT and NT samples compared to pre-OIT samples. When we focused on the genes that are different between the two types of immune tolerance, we observed IL 10RA, IGHG4, CD40, TAP1, IL23A, IL21, CD79A, and CD79B are higher expressed in OIT-induced tolerance. $C D 79 A$ and $C D 79 B$ involved in membrane immunoglobulins to form the B-cell receptor complex ${ }^{65}$. This is an important observation, however the reasons why there is such a difference remain to be elucidated. In contrast, STAT6, FOXP1 NOTCH2, TLR9, AHR, BCL6, CARD11, DOCK11, CD22, PIK3CA are high in NT. Another supportive finding for more innate characteristic and suppressor function of specific B cells in NT and may play a role in long-term tolerance.

We also examined the secreted cytokines and surface receptors from aS ${ }_{1}$-casein-specific $B$ cells. These experiments were essential to characterize specific B cells in more detail. Since there were very low numbers of specific $B$ cells, we had to immortalize and expand these cells. As a key finding of this group of experiments, IL-10 and LAP TGF- $\beta$ were increased in NT and LAP TGF- $\beta$ in OIT-tolerance compared to allergic individuals, demonstrating an important immune regulatory property gained by specific $B$ cells. The majority of secreted proteins displayed characteristics of suppression of inflammation, such as TNFRSF9 marker of tumor-infiltrating regulatory T-cell in lung cancer ${ }^{66}$, AXIN1 dysregulates Wnt pathway in adult eosinophilic esophagitis ${ }^{67}$, uPA modulate airway eosinophilia ${ }^{68}$, STAMBP inhibiting cytokine secretion through the NLRP3 and NLRP7 inflammasomes ${ }^{69,70}$, osteoprotegerin as suppressor of bone resorption and allergic asthma and rheumatoid arthritis ${ }^{71}$, MMP-1 associating with bronchial hyperresponsiveness in asthma ${ }^{72}$, Flt3L cytokine that prevents allergic asthma in mouse model ${ }^{73}, \mathrm{CASP}-$ 8 mediates IL-1 signalling in promoting Th2 responses in asthma ${ }^{74}$, CSF-1 suppresses sensitization to aeroallergens and allergic lung inflammation in asthmatic mice ${ }^{66}$, were increase in NT, suggesting the reduction of inflammation. These molecules are multifunctional, however, their real role in immune tolerance remain to be further analysed. Furthermore, similar increased protein expressions were observed in induced-tolerance after OIT and NT, such as TGF- $\beta$, TNFRSF9, AXIN1, uPA, STAMBP. Some of the proteins were increased in induced tolerance but not in NT. For example, CXCL10, CCL20 that activates Th2 cells and the expression of allergic airway disease ${ }^{75}$. VEGF-A plays a role in allergeninduced nasal inflammation ${ }^{76}$. IL-6, MCP-1 ${ }^{77}$ and MCP-4 ${ }^{78}$ that are associated with asthma susceptibility and severity and MMP-10 that contributes to airway remodelling in asthma ${ }^{79}$. ARTN that causes hypersensitivity in atopic dermatitis ${ }^{80}$. Overall, these data show that although there are similarities in NT and OIT-induced tolerance, there seem to be slightly different underlying mechanisms involved.

In conclusion, we demonstrated a detailed characterization of the transcriptome, secreted proteins, and secreted specific antibodies of allergen-specific B cells in cow's milk OIT-induced tolerance and NT individuals. We demonstrate that allergen-specific $B$ cells show distinctive changes in induction of desensitization as well as induction of tolerance. The transcriptomic changes in specific B cells in OIT- 
induced tolerance are one step further silencing of genes of B cell activation after desensitization. More complete and more numerous gene expression mechanisms of suppression are exhibited in OIT-induced tolerance when compared to desensitized individuals. A proinflammatory environment is observed in allergen-specific B cells in allergic individuals due to type 2 cytokine-related genes. This proinflammatory environment is altered in specific B cells that gain a suppressor capacity after OIT. There are similarities and differences in children that outgrow food allergy compared to OIT-induced tolerance. B cells appear to have one step further differentiation to pre-plasma stage with the expression of more innate immune receptors in NT. Breg cell-related genes are still active in OIT-induced tolerance with higher expression of IGHG4, IL 10 and TGF- $\beta$ genes. Altogether, our data demonstrated that allergen-specific B cells are induced during OIT and natural tolerance and that they have some major gene expression changes that suggest an important role in induction, and maintenance of immune tolerance to food antigens.

\section{Materials And Methods}

\section{Subjects}

A total of twenty-four children with CMA who received oral OIT and children who naturally outgrew cow's milk allergy were recruited for this study. The subjects were assessed by a double-blind placebocontrolled food challenge (DBPCFC) as previously described ${ }^{81,82} 83$. For the CMA group, PBMC were collected and biobanked at 0 months before the immunotherapy, during OIT 6-8 months, and 18-35 months ( $n_{\text {before OIT, during OIT, after OIT }}=9$ ), and 4 additional patients after receiving 18-35 months immunotherapy timepoint $\left(\mathrm{n}_{\text {after OIT }}=4, \mathrm{n}_{\text {after OIT total }}=13\right)$. Within a total of 13 patients from after OIT group, they were characterized as desensitized and tolerance $\left(n_{\text {after OIT (desensitized) }}=6, n_{\text {after OIT (tolerance) }}=7\right.$ ). A group of children who had presented with previous allergic symptoms before and naturally outgrew cow's milk allergy and developed clinical tolerance was also included ( $\left.n_{\text {natural tolerance }(N T)}=9\right)$. PBMC samples were biobanked at the Stanford University Sean Parker Asthma and Allergy Center, California, USA. Each participant with CMA older than 4 years was eligible for inclusion if they had proven sensitivity to milk documented by both a skin prick test greater than $3.5 \mathrm{~mm}$ (wheal) and specific IgE greater than $2 \mathrm{kU} / \mathrm{L}$ to cow's milk extract. Six weeks after the immunotherapy, participants withdrew the treatments and were challenged with cow's milk extract again. The treatment outcomes were defined based on allergic reaction results from cow's milk extract re-challenge at six weeks after the immunotherapy. Patients who showed no reaction up to $32.7 \mathrm{mg}$ dose during the challenge, but began to show reactions at this dose were classified as desensitized. Patients who tolerated the 8,000-12,000 mg dose without any clinical reactions were identified as tolerance patients. Further characteristics of participants are listed in Table 1. All participant materials have been biobanked under the ethical permissions of local institutional ethical committees (Ethical permit number: IRB 8629, Sean N. Parker Center for Allergy \& Asthma Research at Stanford University and Division of Pulmonary, Allergy, and Critical Care Medicine, Stanford University, CA, USA) and the study was conducted following the Helsinki Declaration. As a control, we included 9 healthy individuals between 27-40 years of age who had no allergic symptoms and consumed cow's milk $\left(\mathrm{n}_{\mathrm{HC}}=9\right)$. 


\section{Labelling the allergen}

The purified major allergen $\mathrm{aS}_{1}$-casein from cow's milk was a kind gift from Dr. Els Van Huffen (NIZO food research, Ede, The Netherlands). $\mathrm{aS}_{1}$-casein is a heat-stable and highly allergenic cow's milk allergen. This purified protein was confirmed with the ToxinSensor ${ }^{\mathrm{TM}}$ gel clot endotoxin assay kit (GenScript, NJ, USA) as Lipopolysaccharide (LPS)-free. Afterward, $\mathrm{aS}_{1}$-casein was labeled with a 20 -fold molar excess of biotin reagent that resulted in 4-6 biotin groups per antigen molecule according to manufacturer's instruction (Thermo Fisher Scientific, Waltham, MA, USA). The molar ratio of Sulfo-NHSLC-Biotin to $\mathrm{aS}_{1}$-casein was adjusted with titration experiment. Biotinylated $\mathrm{aS}_{1}$-casein was titrated and used at a concentration of $0.625 \mu \mathrm{g}$ for the labeling of $\mathrm{aS}_{1}$-casein-specific $B$ cells.

\section{Food allergen-specific B cells isolation with flow cytometry}

For the isolation of $\mathrm{aS}_{1}$-casein specific $\mathrm{B}$ cells, biobanked peripheral blood mononuclear cells (PBMCs) were thawed and stained using zombie yellow viability dye (Biolegend, San Diego, CA, USA), washed in staining buffer (PBS, pH 7.2, 0.5\% bovine serum albumin, $2 \mathrm{mM}$ EDTA), and resuspended in $100 \mu \mathrm{L}$ staining buffer. Then, cells were incubated with antibodies against the surface markers CD19 (APC-eflour 780, clone: HIB19, ebioscience, San Diego, CA, USA), CD27 (AF700, clone: M-T271, Biolegend, San Diego, CA, USA), IgG (Dy405, goat-anti-human IgG, Sanquin, Amsterdam, Netherlands), IgA (AF488, goat-antihuman IgA, Jackson Immuno Research Europe Ltd, Cambridgeshire, UK) and IgM (PerCP/Cy5.5, clone: MHM-88, Biolegend, San Diego, CA, USA) at $4^{\circ} \mathrm{C}$ for $15 \mathrm{~min}$. After a washing step, cells were incubated with $0.625 \mathrm{ug} / \mathrm{ml}$ biotinylated $\mathrm{aS}_{1}$-casein and subsequently incubated with the combination of streptavidin PE (BD bioscience, San Jose, CA, USA) and streptavidin Alexa fluor 635 (Thermo Fisher Scientific, Waltham, MA, USA) at $4^{\circ} \mathrm{C}$ for 15 min. aS ${ }_{1}$-casein-specific B cells were gated as ZombieYellow $\mathrm{CD}_{19}{ }^{+} \mathrm{aS}_{1}$-casein-PE${ }^{+}$alexa fluor $635^{+}$and purified using a FACS ARIA III cell sorter (BD bioscience, San Jose, CA, USA). The summary of the antibodies panel used in the experiment is shown in supplementary table 5. The enrichment sorting gate of $\mathrm{aS}_{1}$-casein specific $B$ cells were shown in supplementary Fig 2. Data were analysed with Flowjo, LLC Software (version 10, San Jose, CA, USA).

\section{Immortalisation of food allergen-specific B cells}

Both purified $\mathrm{aS}_{1}$-casein-specific and non-specific $B$ cells were transduced with a retroviral vector containing green fluorescence protein (GFP), BCL6, and BCl-xL (a kind gift from AIMM Therapeutics, Amsterdam, the Netherlands) and cultured in IMDM medium (Thermo Fisher Scientific, Waltham, MA, USA) with CD40L-expressing L cells in the presence of IL-21 for 36 hours. These long-living B cells stably express functional BCR, display a memory phenotype, and produce antibodies and cytokines, thus offering many experimental advantages compared to Epstein-Barr virus (EBV) transformed B cells. ${ }^{84}$ The combination of $B C L-6$ and $B C L-x L$ overexpression and the CD40L/IL-21 culture system was described previously 8586 . 
$5 \mu \mathrm{g} / \mathrm{ml} \mathrm{aS}_{1}$-casein was coated to a Nunc Maxisorb microtiter plate (Thermo Fisher Scientific, Waltham, MA, USA) at $4^{\circ} \mathrm{C}$ overnight and then blocked with blocking buffer (PBS pH 7.4, 5\% BSA, 0.05\% Tween 20). Supernatants from aS ${ }_{1}$-casein-specific $B$ cell samples were added and incubated for 2 hours at room temperature. Specific IgE was detected using a biotinylated anti-human IgE Abs (Kind gift of Dr. C. Heusser, Novartis, Basel, Switzerland), followed by streptavidin-peroxidase (Sigma-Aldrich, St. Louis, MO, USA). For the detection of specific IgG, goat anti-human IgG-peroxidase (Jackson Immuno Research Europe Ltd, Cambridgeshire, UK) was used. For specific IgG1 and IgG4 subclass detection, biotinylated anti-human IgG1 (clone: G17-1 RUO, BD bioscience, San Jose, CA, USA) or biotinylated anti-human IgG4 RJ4 Abs (Abingdon Health, York, UK) were used followed by incubation with streptavidin-peroxidase conjugated (Sigma-Aldrich, St. Louis, MO, USA). For specific IgG2 and IgG3 subclass detections, mouse anti-human IgG2 (clone: MH162-1, HP 6014, Sanquin, Amsterdam, the Netherlands) and mouse antihuman IgG3 (clone: MH163-1, HP 6095, Sanquin, Amsterdam, the Netherlands) were labeled with biotin (Sigma-Aldrich, St. Louis, MO, USA) and used as the primary detection antibodies. For specific IgA1 and IgA2 subclass detections, mouse anti-human IgA1 (clone: B3506B4, Abcam, Cambridge, UK) and mouse anti-human IgA2 (clone: B3506B4, Abcam, Cambridge, UK) were labelled with biotin (Sigma-Aldrich, St. Louis, MO, USA) and used as the primary detection antibodies. Goat anti-Human IgD Antibody HRP Conjugated (clone: A80-106P, Bethyl Laboratories, Montgomery, TX, USA) was used for the detection of specific IgD. ELISAs were developed using tetramethylbenzidine (TMB) substrate (Thermo Fisher Scientific, Waltham, MA, USA) and the reaction was stopped with $1 \mathrm{M} \mathrm{H}_{2} \mathrm{SO}_{4}$ sulfuric acid at OD450 nm. Plates were read at $450 \mathrm{~nm}$ by a Mithras LB 940 spectrophotometer (Berthold Technologies, Bad Wildbad, Germany).

\section{Library preparation and next generation of ultra-low input RNA-sequencing}

Next generation RNA-sequencing library development includes cell lysis, reverse transcription into the first-strand cDNA, second-strand synthesis, and cDNA amplification. Sorted $a S_{1}$-casein-specific $B$ cells of children with cow's milk allergy before OIT, after OIT, and children who spontaneously outgrew food allergy were lysed. Purified total RNA (100 ng) was ribosome depleted and then reverse-transcribed into double-stranded cDNA, with actinomycin added during first-strand synthesis. cDNA samples were fragmented, end-repaired, and polyadenylated before ligation using the SMART-seq ${ }^{\circledR}$ V4 ultra ${ }^{\circledR}$ low input RNA kit (Takara Bio, Mountain View, CA, USA). The cDNA was amplified with 11 cycles of PCR. To achieve the highest purity of CDNA, PCR clean-up was performed using Agencourt AMPure XP beads (Beckman Coulter $\mathrm{GmbH}$, Krefeld, Germany). The quality and quantity of the cDNA were determined with a Qubit 1.0 Fluorometer (Life Technologies, South San Francisco, Calif) and the Bioanalyser 2100 (Agilent Technologies, Waldbronn, Germany). Then, the Illumina Nextera XT kit (Nextera XT DNA Library Preparation Kit (Illumina, San Diego, CA) was used to make cDNA libraries suitable for Illumina sequencing. The libraries were normalized to $10 \mathrm{nM}$ in Tris-Cl $10 \mathrm{mM}, \mathrm{pH} 8.5$ with $0.1 \%$ Tween 20. After that, sequencing was performed on the Nova seq (Illumina Way, San Diego, CA, USA). The sequencing results were quantified in read-based terminologies, such as RPKM/FPKM (read/fragment per kilobase 
per million mapped reads) and normalized counts at the Functional Genomic Center Zurich, University of Zurich, Switzerland.

\section{RNA-sequencing data analysis}

The quality control on raw sequence data was analysed with FastQC (version 0.10.0; Babraham Institute, Cambridge, United Kingdom) and mapped to the Homo sapiens genome (GRCh38 build) using RSEM (version 1.2.12) implementation of the Bowtie software (version 1.0.0) alignment program with the Ensembl annotation (version 75). Gene and isoform level abundances were quantified as reads per kilobase per million mapped read values. Clustering analyses were performed with the "ward.D2" clustering algorithm implemented in the "hclust" function of R statistics package. Heat map plots were performed with the function "heatmap.2" implemented in the gplots R package. Differential expression analysis between the 2 groups was performed with the edgeR Bioconductor package. Genes present in less than $75 \%$ of samples in both conditions were excluded. Q-values were calculated by using the Benjamini-Hochberg method, and genes with a q-value of less than 0.015 and an absolute log2 (fold change) value of greater than 1 were kept for further analysis. Gene Ontology term enrichment analysis was performed with the GOseq Bioconductor package using the Wallenius approximation. Pathway analysis was done with MetaCore version 6.3 (Thomson Reuters) Enricher platform with the Panther database. Significant pathways were defined as pathways with an adjusted p-value of less than .01 . Gene lists were created as described in the text or based on gene lists from NIAID ImmPort Resources (http://www.immport.org/immport-open/public/reference/genelists). Genes with p-value $<=0.01$ and log2 fold change $>=0.5$ were used in this study. Gene network enrichment was generated using MetaCore version 6.3 (Thomson Reuters), and identification of shared genes was analysed by Venn diagram tool (FGCZ VennTool, functional genomics center Zurich, Switzerland). Data was uploaded to GEO database as PRJEB48819.

\section{Proximity extension assay (PEA)}

The PEA with Olink inflammatory panel (Olink Biosciences, Uppsala, Sweden) used in this study includes 92 proteins and for each sample, $1 \mu \mathrm{L}$ of cultured B cell supernatants was used. The PEA method is achieved by the binding of PEA probes on paired antibodies from the samples. The first step is the immune reaction in which 92 Abs pairs, labeled with DNA oligonucleotides, bind to their respective protein in the samples during 16-22 hours incubation. Next, extension and pre-amplification were performed in which oligonucleotides were extended using a DNA polymerase. This newly created piece of DNA barcode is amplified by PCR for 2 hours. Then, detection in which the amount of each DNA barcode is quantified by microfluidic qPCR in 4 and a half hours. Finally, the detection of proteins of interest was examined through quantitative real-time PCR. The PCR results were evaluated as normalized protein expression (NPX) values, which are arbitrary units on a $\log _{2}$ ratio. NPX is calculated from Ct values and data preprocessing is performed to minimize both intra- and inter-assay variation. A high NPX value corresponds to a high protein concentration and expresses relative quantification between samples but represents no absolute quantification. Assay characteristics including quality control, detection limits, and 
measurements of assay performance and validation were done at the Olink reference center, the Swiss Institute of Allergy and Asthma Research (SIAF), Davos, Switzerland.

\section{Statistical analysis}

Data were expressed as a mean \pm standard error of the mean (SEM). Statistical analysis was conducted using GraphPad Prism 7.0 Software (GraphPad Software, La Jolla, CA, USA). Gene expression between multiple groups was analysed by using the Wilcoxon signed-ranked test, and comparison between groups was done with the Mann-Whitney test. A p-value of less than 0.05 was considered statistically significant.

\section{Declarations}

\section{Author contributions}

P.S. carried out the experiments of the study, data analysis, and manuscript preparation. W.V. and M.A. provided critical inputs in designing the study and interpretation of results. G.T. conducted a statistical analysis approach to RNA-seq data. P.S., W.V., G.T., O.F.W., K.J., M.S., S.D.B., C.A.A., K.N., M.A. contributed to the data analysis and interpretation of the results. D.M., A.G., T.B., S.R.S. assisted in performing the experiments. E.B. analysed the proximity extension data. H.S. provided knowledge regarding the immortalisation technique of allergen-specific B cells. I.C. recruited the participants and biobanked the PBMCs specimens. M.A. supervised the project. All authors contributed to the revision of the manuscript.

\section{Declaration of research fundings}

M.A. has received research support from the Swiss National Science Foundation No. 310030201053/320030-159870 and the Sean N Parker Center for Allergy and Asthma Research at Stanford University. C.A.A. is employed by the Swiss Institute of Allergy and Asthma Research, University of Zurich; has research grants from the Swiss National Science Foundation No. 310030-156823, and the Christine Kühne - Center for Allergy Research and Education (CK-CARE). W.V. received research grant from Promedica stiftung. M.S. received research grants from the Swiss National Science Foundation (No. 310030_189334/1) and the GSK, and Novartis. K.N. has received the funding from NIAID grants U19Al104209, U01Al140498, and R01Al140134 and the Sean N. Parker Center for Allergy and Asthma Research at Stanford University.

\section{Acknowledgments}

We thank all of the staff members of the Swiss Institute of Allergy and Asthma Research (SIAF) for their support, Beate Rückert for her help with flow cytometry, Patrick Westermann for his contribution to protein component detection, Anja Heider for her help with the OLINK measurement and Juan Rodriguez Coira for his advice of the partial interpretation of RNA-seq results. We also thank to Dr. Els Van Huffen at NIZO food research, the Netherlands, for providing us the purified major allergen $\mathrm{aS}_{1}$-casein. 
C.A.A reports research grants from Allergopharma, Idorsia, GlaxoSmithKline, Novartis Research Institutes, Astra Zeneca, Swiss National Science Foundation, Christine Kühne-Center for Allergy Research and Education, grant from European Commission's Horison's 2020 Framework Programme, Cure, advisory role in Sanofi/Regeneron, and Scibase, Sweden. M.S. reports research grants from the Swiss National Science Foundation (No. 310030_189334/1), GSK and Novartis and speaker's fee from AstraZeneca. S.D.B. has consulted for Regeneron, Sanofi, and Novartis on topics unrelated to this study, owns stock in AbCellera Biologics, has received grant support from the U.S. National Institutes of Health and the Bill and Melinda Gates Foundation, and has an endowment from the Crown family foundation.

\section{Additional information}

Supplementary information is available for this paper.

\section{Abbreviations}

Abs Antibodies

CD Cluster of differentiation

CD40L CD40 ligand

CMA Cow's milk allergy

cDNA Complementary DNA

Bcl-6 B-cell lymphoma 6

Bcl-xl B-cell lymphoma-extra large

BCR B cell receptor

Breg B Regulatory

DEGs Differentially expressed genes

OIT Oral allergen-specific immunotherapy

GFP Green fluorescence protein

$\mathrm{HC} \quad$ Healthy controls

Igs Immunoglobulins

IL Interleukin

LPS Lipopolysaccharide 
PBMCs Peripheral blood mononuclear cells

PEA Proximity extension assay

RNA Ribonucleic acid

RNA-seq RNA-sequencing

slgE Specific immunoglobulin E

slgG Specific immunoglobulin G

slgG1 Specific immunoglobulin G1

slgG4 Specific immunoglobulin G4

TGF- $\beta \quad$ Transforming growth factor beta

TLR Toll-like receptor

\section{References}

1. Gupta RS, et al. The Public Health Impact of Parent-Reported Childhood Food Allergies in the United States. Pediatrics 142, (2018).

2. Sicherer SH, Sampson HA. Food allergy: A review and update on epidemiology, pathogenesis, diagnosis, prevention, and management. J Allergy Clin Immunol 141, 41-58 (2018).

3. Flom JD, Sicherer SH. Epidemiology of Cow's Milk Allergy. Nutrients 11, (2019).

4. Lifschitz C, Szajewska H. Cow's milk allergy: evidence-based diagnosis and management for the practitioner. Eur J Pediatr 174, 141-150 (2015).

5. Nurmatov U, et al. Allergen immunotherapy for IgE-mediated food allergy: a systematic review and meta-analysis. Allergy 72, 1133-1147 (2017).

6. Globinska A, et al. Mechanisms of allergen-specific immunotherapy: Diverse mechanisms of immune tolerance to allergens. Ann Allergy Asthma Immunol 121, 306-312 (2018).

7. Satitsuksanoa P, Jansen K, Globinska A, van de Veen W, Akdis M. Regulatory Immune Mechanisms in Tolerance to Food Allergy. Front Immuno/ 9, 2939 (2018).

8. Boyce JA, et al. Guidelines for the diagnosis and management of food allergy in the United States: summary of the NIAID-sponsored expert panel report. Nutr Res 31, 61-75 (2011). 
9. Satitsuksanoa P, Daanje M, Akdis M, Boyd SD, van de Veen W. Biology and dynamics of B cells in the context of IgE-mediated food allergy. Allergy, (2020).

10. Satitsuksanoa P, van de Veen W, Akdis M. B cells in food allergy. J Allergy Clin Immuno/ 147, 49-51 (2021).

11. Muraro A, et al. EAACl food allergy and anaphylaxis guidelines: diagnosis and management of food allergy. Allergy 69, 1008-1025 (2014).

12. Jimenez-Saiz R, et al. IgG1(+) B-cell immunity predates IgE responses in epicutaneous sensitization to foods. Allergy 74, 165-175 (2019).

13. Karlsson MR, Rugtveit J, Brandtzaeg P. Allergen-responsive CD4+CD25+ regulatory $\mathrm{T}$ cells in children who have outgrown cow's milk allergy. J Exp Med 199, 1679-1688 (2004).

14. Hoh RA, et al. Origins and clonal convergence of gastrointestinal $\lg \mathrm{E}(+) \mathrm{B}$ cells in human peanut allergy. Sci Immunol 5, (2020).

15. Croote D, Darmanis S, Nadeau KC, Quake SR. High-affinity allergen-specific human antibodies cloned from single lgE B cell transcriptomes. Science 362, 1306-1309 (2018).

16. Heeringa JJ, et al. IgE-expressing memory B cells and plasmablasts are increased in blood of children with asthma, food allergy, and atopic dermatitis. Allergy 73, 1331-1336 (2018).

17. Gupta J, et al. Resolving the etiology of atopic disorders by using genetic analysis of racial ancestry. J Allergy Clin Immunol 138, 676-699 (2016).

18. Shan M, et al. Secreted IgD Amplifies Humoral T Helper 2 Cell Responses by Binding Basophils via Galectin-9 and CD44. Immunity 49, 709-724 e708 (2018).

19. Wal JM. Cow's milk proteins/allergens. Ann Allergy Asthma Immuno/ 89, 3-10 (2002).

20. Wal JM. Bovine milk allergenicity. Ann Allergy Asthma Immunol 93, S2-11 (2004).

21. Hoh RA, et al. Single B-cell deconvolution of peanut-specific antibody responses in allergic patients. J Allergy Clin Immunol 137, 157-167 (2016).

22. van de Veen $\mathrm{W}$, et al. IgG4 production is confined to human IL-10-producing regulatory $\mathrm{B}$ cells that suppress antigen-specific immune responses. J Allergy Clin Immunol 131, 1204-1212 (2013).

23. Boonpiyathad $\mathrm{T}$, et al. Role of Der $\mathrm{p}$ 1-specific $\mathrm{B}$ cells in immune tolerance during 2 years of house dust mite-specific immunotherapy. J Allergy Clin Immunol 143, 1077-1086 e1010 (2019).

24. Okamoto $S$, et al. Predictive value of IgE/lgG4 antibody ratio in children with egg allergy. Allergy Asthma Clin Immunol 8, 9 (2012). 
25. Cerecedo I, et al. Mapping of the IgE and IgG4 sequential epitopes of milk allergens with a peptide microarray-based immunoassay. J Allergy Clin Immunol 122, 589-594 (2008).

26. Boonpiyathad T, et al. High-dose bee venom exposure induces similar tolerogenic B-cell responses in allergic patients and healthy beekeepers. Allergy 72, 407-415 (2017).

27. Caubet JC, et al. Natural tolerance development in cow's milk allergic children: IgE and IgG4 epitope binding. Allergy 72, 1677-1685 (2017).

28. Ranasinghe R, Eri R. Modulation of the CCR6-CCL20 Axis: A Potential Therapeutic Target in Inflammation and Cancer. Medicina (Kaunas) 54, (2018).

29. Jutel M, Jaeger L, Suck R, Meyer H, Fiebig H, Cromwell O. Allergen-specific immunotherapy with recombinant grass pollen allergens. J Allergy Clin Immunol 116, 608-613 (2005).

30. Jutel M, et al. IL-10 and TGF-beta cooperate in the regulatory $\mathrm{T}$ cell response to mucosal allergens in normal immunity and specific immunotherapy. Eur J Immunol 33, 1205-1214 (2003).

31. Cevhertas $\mathrm{L}$, et al. IL-10 induces IgG4 production in NOD-scid II2rgamma(null) mice humanized by engraftment of peripheral blood mononuclear cells. Allergy, (2021).

32. Suzuki M, et al. Inhibition of allergic responses by CD40 gene silencing. Allergy $64,387-397$ (2009).

33. Kim JH, et al. Genetic association analysis of TAP1 and TAP2 polymorphisms with aspirin exacerbated respiratory disease and its FEV1 decline. J Hum Genet 56, 652-659 (2011).

34. Ubel $\mathrm{C}$, et al. The activating protein 1 transcription factor basic leucine zipper transcription factor, ATF-like (BATF), regulates lymphocyte- and mast cell-driven immune responses in the setting of allergic asthma. J Allergy Clin Immunol 133, 198-206 e191-199 (2014).

35. Eder W, et al. Toll-like receptor 2 as a major gene for asthma in children of European farmers. $J$ Allergy Clin Immunol 113, 482-488 (2004).

36. Ayuso $\mathrm{P}$, et al. Variability in histamine receptor genes $\mathrm{HRH} 1, \mathrm{HRH} 2$ and $\mathrm{HRH} 4$ in patients with hypersensitivity to NSAIDs. Pharmacogenomics 14, 1871-1878 (2013).

37. Lawrence MG, et al. Elevated IgE and atopy in patients treated for early-onset ADA-SCID. J Allergy Clin Immunol 132, 1444-1446 (2013).

38. Sicherer SH, Bollinger ME, Hershfield MS, Sampson HA, Lederman HM. Food allergy in a patient with adenosine deaminase deficiency undergoing enzyme replacement with polyethylene glycol-modified adenosine deaminase. J Allergy Clin Immuno/ 101, 561-562 (1998). 
39. Yeon SM, et al. IL-7 plays a critical role for the homeostasis of allergen-specific memory CD4 T cells in the lung and airways. Sci Rep 7, 11155 (2017).

40. Meyts I, et al. IL-12 contributes to allergen-induced airway inflammation in experimental asthma. $J$ Immuno/ 177, 6460-6470 (2006).

41. Sanders NL, Mishra A. Role of interleukin-18 in the pathophysiology of allergic diseases. Cytokine Growth Factor Rev 32, 31-39 (2016).

42. Peng J, Yang XO, Chang SH, Yang J, Dong C. IL-23 signaling enhances Th2 polarization and regulates allergic airway inflammation. Cell Res 20, 62-71 (2010).

43. Campbell JD, Gangur V, Simons FE, HayGlass KT. Allergic humans are hyporesponsive to a CXCR3 ligand-mediated Th1 immunity-promoting loop. FASEB J 18, 329-331 (2004).

44. Ohta T, et al. Crucial roles of XCR1-expressing dendritic cells and the XCR1-XCL1 chemokine axis in intestinal immune homeostasis. Sci Rep 6, 23505 (2016).

45. Otero K, et al. Nonredundant role of CCRL2 in lung dendritic cell trafficking. Blood 116, 2942-2949 (2010).

46. Tschopp CM, et al. Granzyme B, a novel mediator of allergic inflammation: its induction and release in blood basophils and human asthma. Blood 108, 2290-2299 (2006).

47. Weissler KA, Frischmeyer-Guerrerio PA. Genetic evidence for the role of transforming growth factorbeta in atopic phenotypes. Curr Opin Immunol 60, 54-62 (2019).

48. Ibrahim S, et al. PIK3R3 promotes chemotherapeutic sensitivity of colorectal cancer through PIK3R3/NF-kB/TP pathway. Cancer Biol Ther 19, 222-229 (2018).

49. Honjo A, et al. Pharmacologic inhibition of Notch signaling suppresses food antigen-induced mucosal mast cell hyperplasia. J Allergy Clin Immunol 139, 987-996 e910 (2017).

50. Lechner M, et al. Notch2-mediated plasticity between marginal zone and follicular B cells. Nat Commun 12, 1111 (2021).

51. Iwahashi S, et al. Notch2 regulates the development of marginal zone B cells through Fos. Biochem Biophys Res Commun 418, 701-707 (2012).

52. van Keimpema M, et al. The forkhead transcription factor FOXP1 represses human plasma cell differentiation. Blood 126, 2098-2109 (2015).

53. Berglund LJ, et al. IL-21 signalling via STAT3 primes human naive B cells to respond to IL-2 to enhance their differentiation into plasmablasts. Blood 122, 3940-3950 (2013). 
54. Ward-Kavanagh LK, Lin WW, Sedy JR, Ware CF. The TNF Receptor Superfamily in Co-stimulating and Co-inhibitory Responses. Immunity 44, 1005-1019 (2016).

55. Browne EP. Regulation of B-cell responses by Toll-like receptors. Immunology 136, 370-379 (2012).

56. Clark EA, Giltiay NV. CD22: A Regulator of Innate and Adaptive B Cell Responses and Autoimmunity. Front Immuno/ 9, 2235 (2018).

57. Orgel KA, et al. Exploiting CD22 on antigen-specific B cells to prevent allergy to the major peanut allergen Ara h 2. J Allergy Clin Immunol 139, 366-369 e362 (2017).

58. Avery DT, et al. Germline-activating mutations in PIK3CD compromise B cell development and function. J Exp Med 215, 2073-2095 (2018).

59. Ogasawara T, et al. Allergic TH2 Response Governed by B-Cell Lymphoma 6 Function in Naturally Occurring Memory Phenotype CD4(+) T Cells. Front Immuno/ 9, 750 (2018).

60. Wei Z, et al. Pathogenic CARD11 mutations affect B cell development and differentiation through a noncanonical pathway. Sci Immunol 4, (2019).

61. Ma CA, et al. Germline hypomorphic CARD11 mutations in severe atopic disease. Nat Genet 49, 1192-1201 (2017).

62. Buchbinder D, et al. Mild B-cell lymphocytosis in patients with a CARD11 C49Y mutation. $J$ Allergy Clin Immunol 136, 819-821 e811 (2015).

63. Boonpiyathad T, Pradubpongsa P, Mitthamsiri W, Satitsuksanoa P, Jacquet A, Sangasapaviliya A. Allergen-specific immunotherapy boosts allergen-specific IgD production in house dust mite-sensitized asthmatic patients. Allergy 75, 1457-1460 (2020).

64. Blumchen $\mathrm{K}$, et al. Oral peanut immunotherapy in children with peanut anaphylaxis. J Allergy Clin Immunol 126, 83-91 e81 (2010).

65. Reth M. B cell antigen receptors. Curr Opin Immuno/ 6, 3-8 (1994).

66. Moon HG, et al. Colony-stimulating factor 1 and its receptor are new potential therapeutic targets for allergic asthma. Allergy 75, 357-369 (2020).

67. Giannetti M, Schroeder HA, Zalewski A, Gonsalves N, Bryce PJ. Dysregulation of the Wnt pathway in adult eosinophilic esophagitis. Dis Esophagus 28, 705-710 (2015).

68. Brooks AM, Bates ME, Vrtis RF, Jarjour NN, Bertics PJ, Sedgwick JB. Urokinase-type plasminogen activator modulates airway eosinophil adhesion in asthma. Am J Respir Cell Mol Bio/ 35, 503-511 (2006). 
69. Bednash JS, Johns F, Patel N, Smail TR, Londino JD, Mallampalli RK. The deubiquitinase STAMBP modulates cytokine secretion through the NLRP3 inflammasome. Cell Signal 79, 109859 (2021).

70. Bednash JS, et al. Targeting the deubiquitinase STAMBP inhibits NALP7 inflammasome activity. Nat Commun 8, 15203 (2017).

71. Remuzgo-Martinez S, et al. Expression of osteoprotegerin and its ligands, RANKL and TRAIL, in rheumatoid arthritis. Sci Rep 6, 29713 (2016).

72. Naveed SU, et al. Matrix Metalloproteinase-1 Activation Contributes to Airway Smooth Muscle Growth and Asthma Severity. Am J Respir Crit Care Med 195, 1000-1009 (2017).

73. Agrawal DK, Hopfenspirger MT, Chavez J, Talmadge JE. Flt3 ligand: a novel cytokine prevents allergic asthma in a mouse model. Int Immunopharmacol 1, 2081-2089 (2001).

74. Qi X, et al. Critical role of caspase-8-mediated IL-1 signaling in promoting Th2 responses during asthma pathogenesis. Mucosal Immunol 10, 128-138 (2017).

75. Weckmann M, et al. Critical link between TRAIL and CCL2O for the activation of TH2 cells and the expression of allergic airway disease. Nature medicine 13, 1308-1315 (2007).

76. Choi GS, et al. Vascular endothelial growth factor in allergen-induced nasal inflammation. Clin Exp Allergy 39, 655-661 (2009).

77. Szalai C, et al. Polymorphism in the gene regulatory region of MCP-1 is associated with asthma susceptibility and severity. J Allergy Clin Immunol 108, 375-381 (2001).

78. Lamkhioued B, et al. Monocyte chemoattractant protein (MCP)-4 expression in the airways of patients with asthma. Induction in epithelial cells and mononuclear cells by proinflammatory cytokines. Am J Respir Crit Care Med 162, 723-732 (2000).

79. Kuo CS, et al. Contribution of airway eosinophils in airway wall remodeling in asthma: Role of MMP-10 and MET. Allergy 74, 1102-1112 (2019).

80. Murota $\mathrm{H}$, et al. Artemin causes hypersensitivity to warm sensation, mimicking warmth-provoked pruritus in atopic dermatitis. J Allergy Clin Immunol 130, 671-682 e674 (2012).

81. Begin $\mathrm{P}$, et al. Safety and feasibility of oral immunotherapy to multiple allergens for food allergy. Allergy Asthma Clin Immunol 10, 1 (2014).

82. Begin $\mathrm{P}$, et al. Phase 1 results of safety and tolerability in a rush oral immunotherapy protocol to multiple foods using Omalizumab. Allergy Asthma Clin Immunol 10, 7 (2014).

83. Andorf S, et al. A Phase 2 Randomized Controlled Multisite Study Using Omalizumab-facilitated Rapid Desensitization to Test Continued vs Discontinued Dosing in Multifood Allergic Individuals. 
EClinicalMedicine 7, 27-38 (2019).

84. Kwakkenbos MJ, et al. Generation of stable monoclonal antibody-producing B cell receptorpositive human memory B cells by genetic programming. Nature medicine 16, 123-128 (2010).

85. Kwakkenbos MJ, van Helden PM, Beaumont T, Spits H. Stable long-term cultures of self-renewing B cells and their applications. Immunol Rev 270, 65-77 (2016).

86. van de Veen $\mathrm{W}$, et al. A novel proangiogenic $\mathrm{B}$ cell subset is increased in cancer and chronic inflammation. Sci Adv 6, eaaz3559 (2020).

\section{Table 1}

Table 1 is available in the Supplementary Files section.

\section{Figures}




\section{A}

Before OIT

After OIT
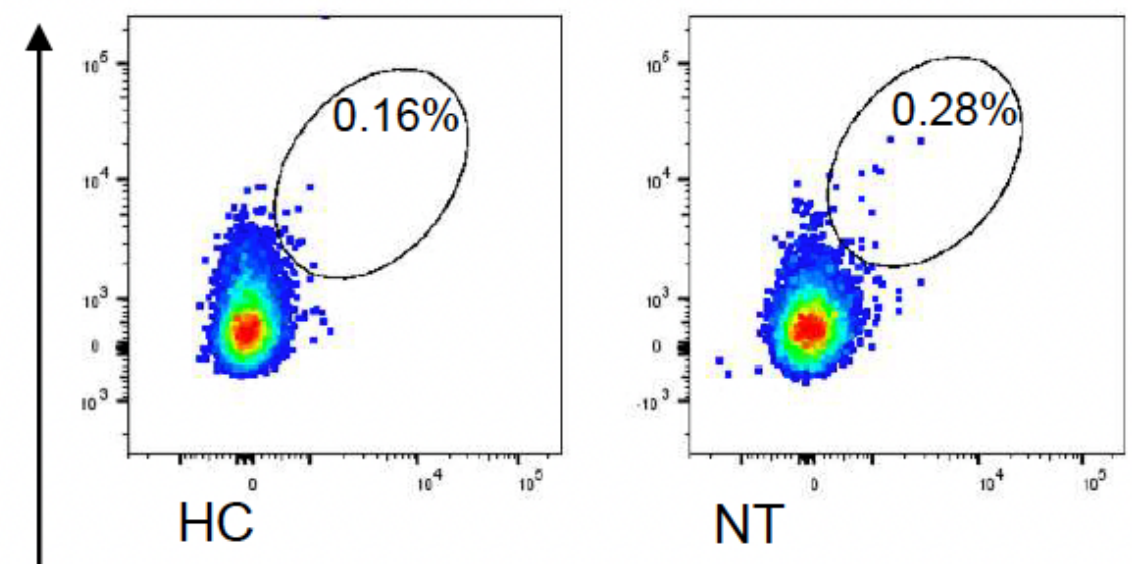

NT
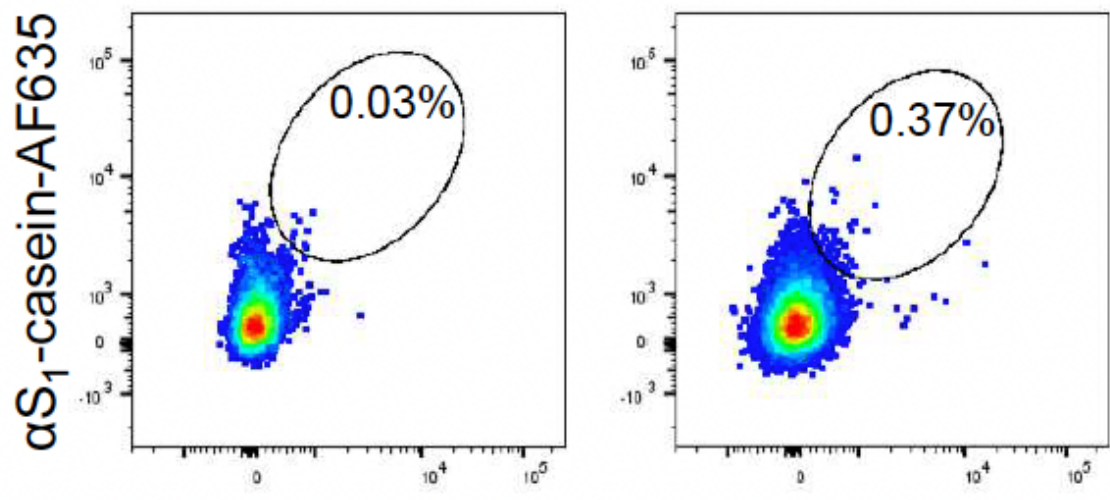

$\alpha S_{1}$-casein-PE

B

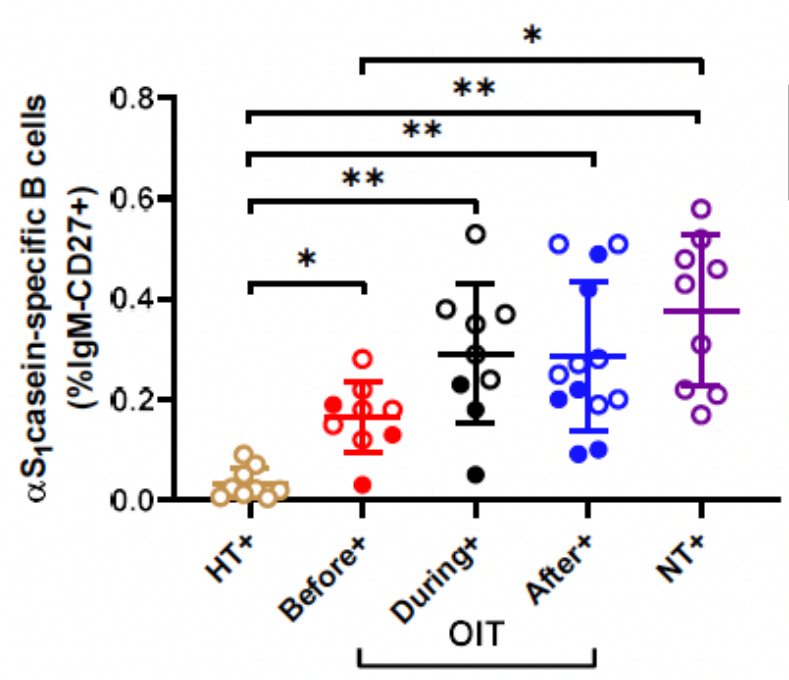

- Deșensitized

O Tolerized

Figure 1

The frequency of aS $\mathrm{S}_{1}$-casein-specific $\mathrm{B}$ cells is increased after OIT in allergic patients and natural tolerance compared to healthy individuals.

Flow cytometry plots in (A) are representative data coming from 4 samples; before OIT, after OIT, HC, and $N T$, respectively. (B) Frequencies of $\mathrm{aS}_{1}$-casein-specific $B$ cells was analysed by one-way ANOVA, ${ }^{*}<0.05$, 

empty dots represent "tolerance" $\left(n_{H C}=9, n_{\text {before OIT }}=9, n_{\text {during OIT }}=9, n_{\text {after OIT }}=13, n_{\text {natural tolerance (NT) }}=9\right.$ )
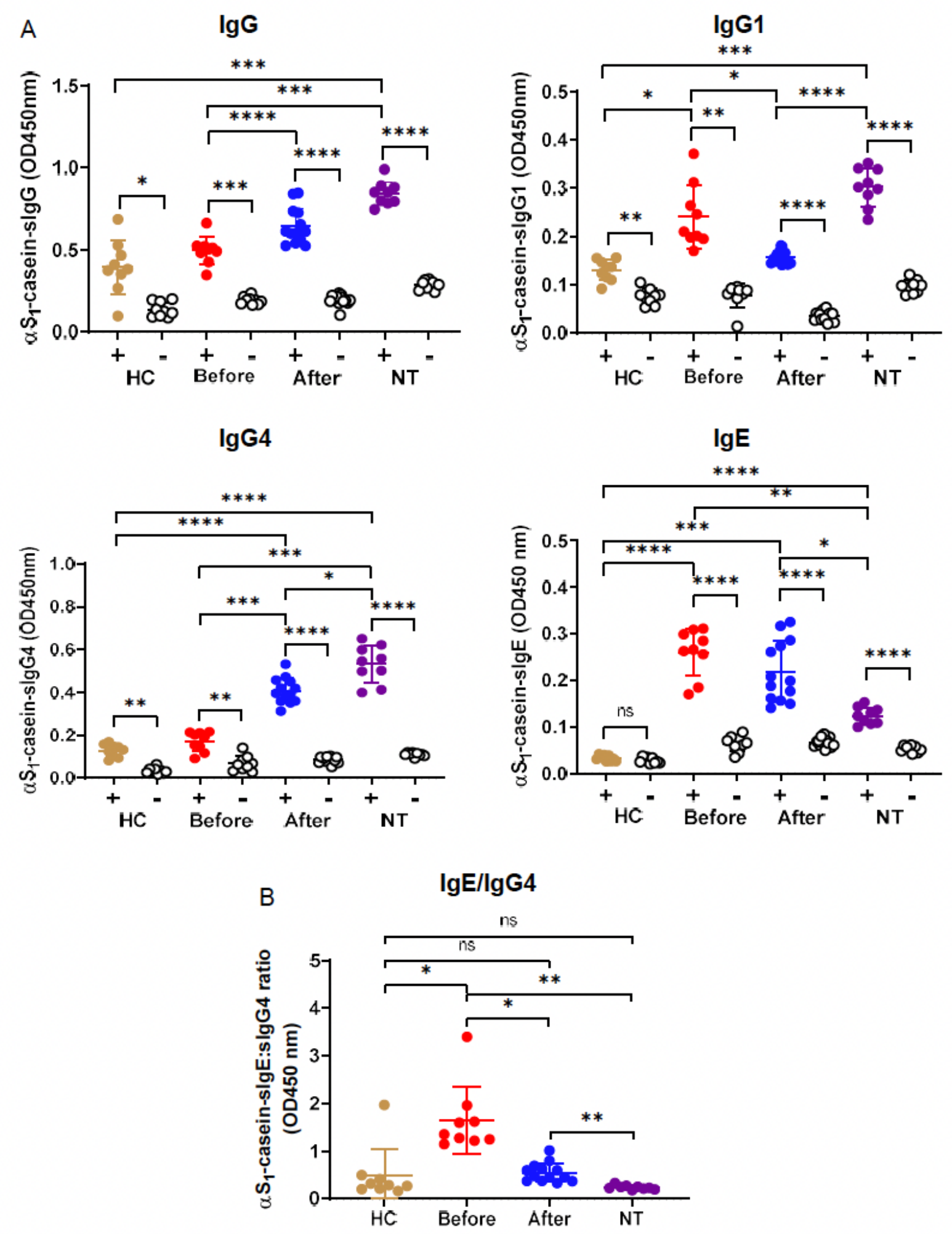

Figure 2 
Specificity of $a S_{1}$-casein-specific immunoglobulin detection in allergic patients undergoing OIT versus natural tolerance and healthy controls.

(A) Detection of $\mathrm{aS}_{1}$-casein-specific immunoglobulins in supernatants of $\mathrm{aS}_{1}$-casein-specific and non-

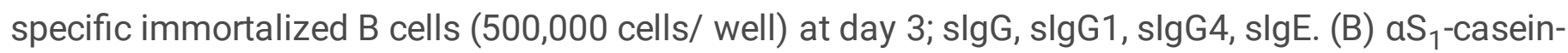
specific slgE/slgG4 ratio analysed by one-way ANOVA, * $p<0.05, * * p<0.01, * \star * p<0.001, * \star \star \star x<0.0001$; Values are means $\pm S E M,\left(n_{H C}=9, n_{\text {before oIT }}=9, n_{\text {after oIT }}=13, n_{\text {natural tolerance }(N T)}=9\right)$. 


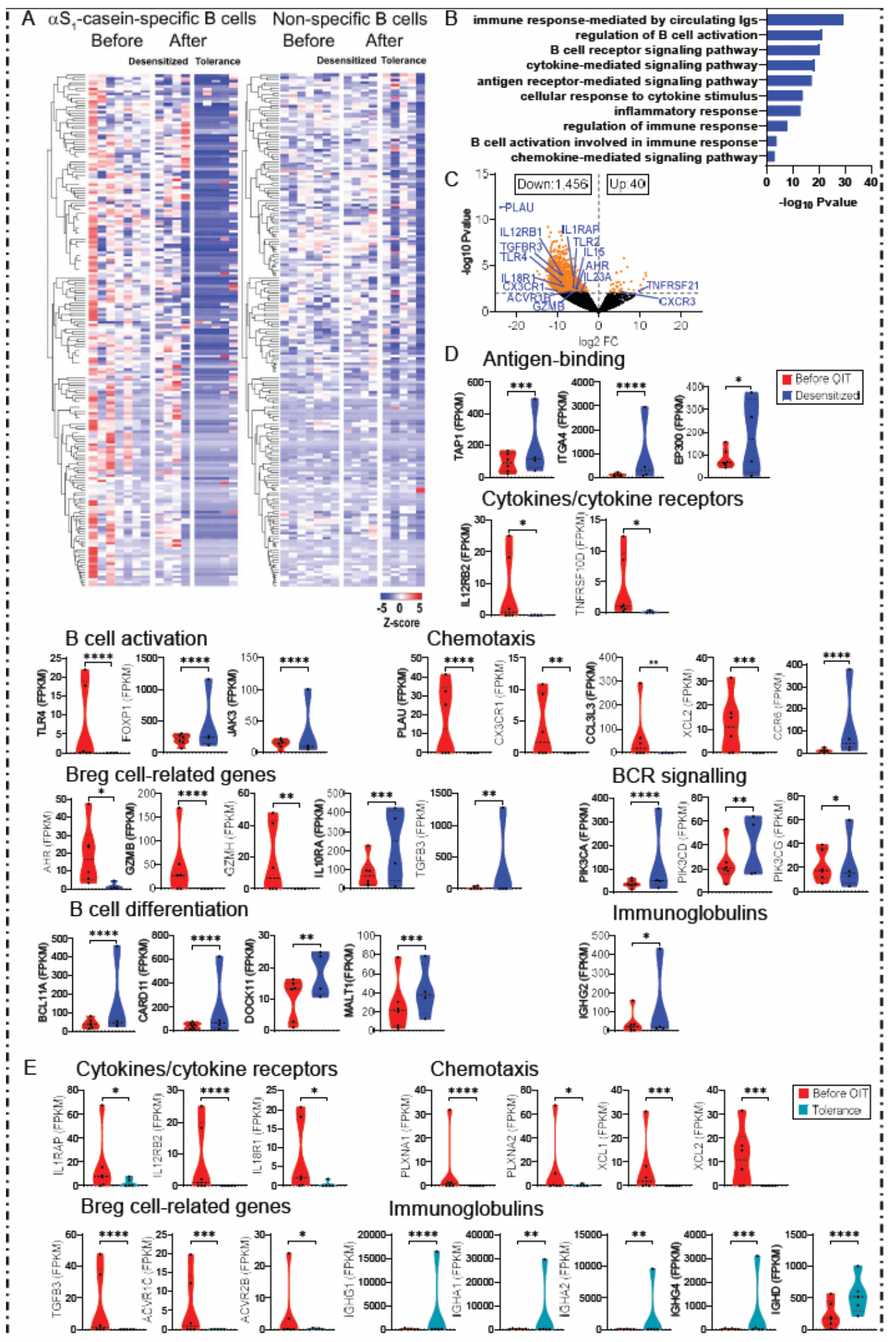

Figure 3

DGEs signatures in $\mathrm{aS}_{1}$-casein-specific and non-specific B cells before and after OIT (desensitized \& tolerance).

(A) Heatmap of the top 200 most DGEs in aS ${ }_{1}$-casein-specific and non-specific $B$ cells from before and after OIT, $\mathrm{n}_{\text {before OIT }}=6, \mathrm{n}_{\text {after OIT (desensitized) }}=4, \mathrm{n}_{\text {after OIT (tolerance) }}=5$ (B) Top B cell-related pathways analysis 
(C) Volcano plot shows differentially expressed genes with top 20 significant genes ( $p$-value $<0.05$ ). Violin plots of differentially expressed genes in 8 different gene groups; antigen-binding, cytokines/ cytokine receptors, $B$ cell activation, chemotaxis, Breg cell-related genes, BCR signalling, B cell differentiation, and immunoglobulins; (D) before OIT vs desensitized and (E) before OIT vs tolerance, * $p<0.05$, $* \star p<0.01$, *** $p<0.001, * \star \star \star p<0.0001$.

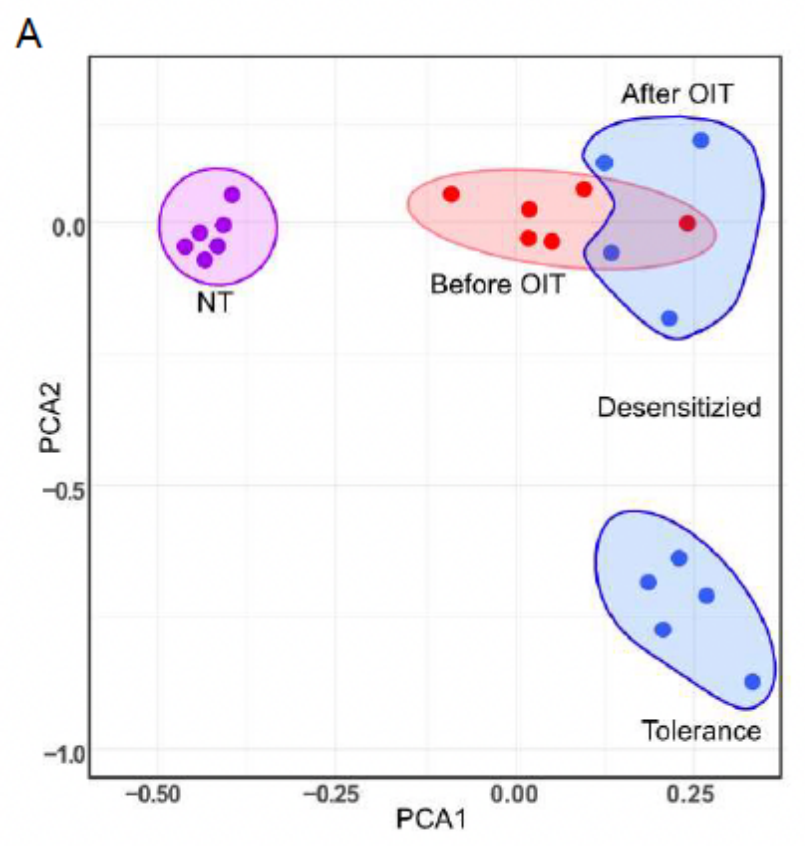

B $\quad \alpha \mathrm{S}_{1}$-casein-specific $B$ cells

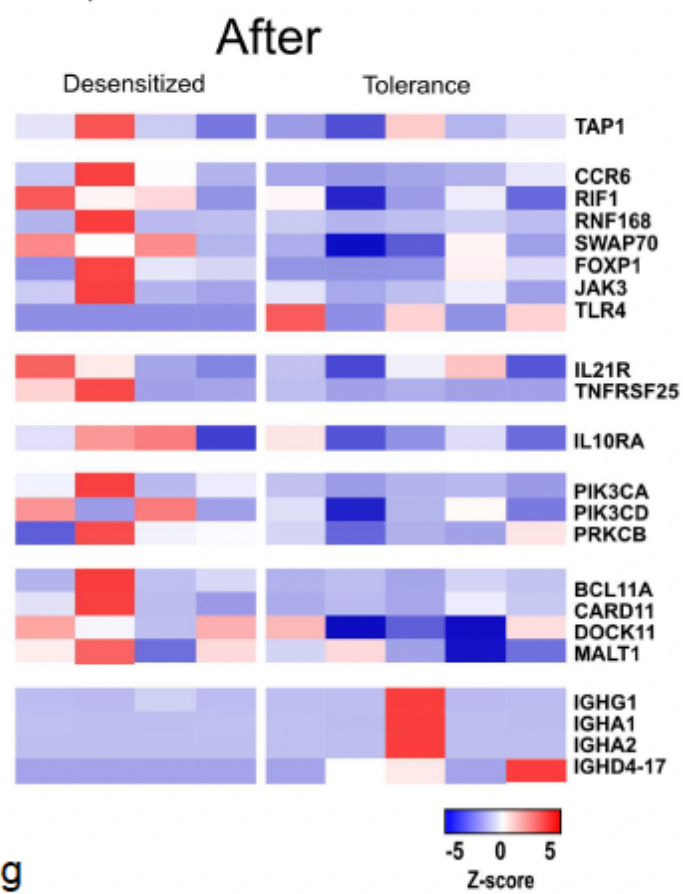

C Antigen-binding

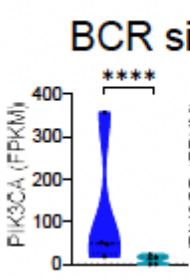

signalling
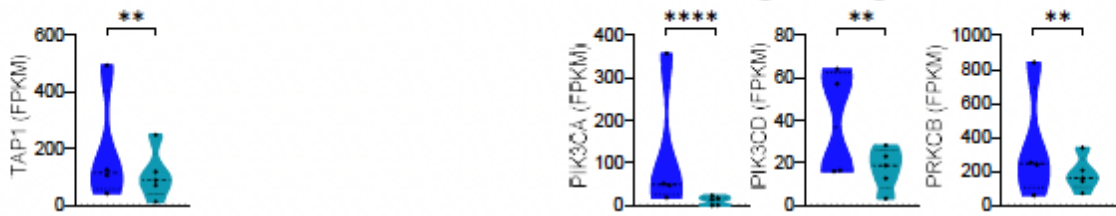

B cell activation
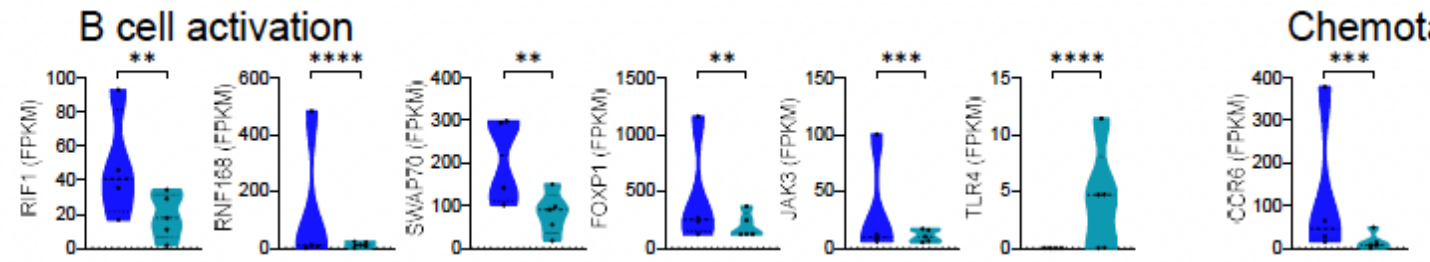

Cytokines/cytokine receptors

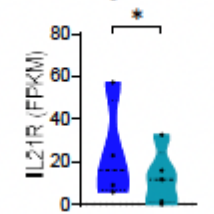

Breg cell-related genes

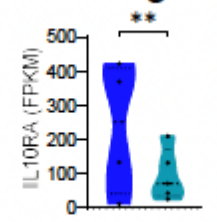

B cell differentiation
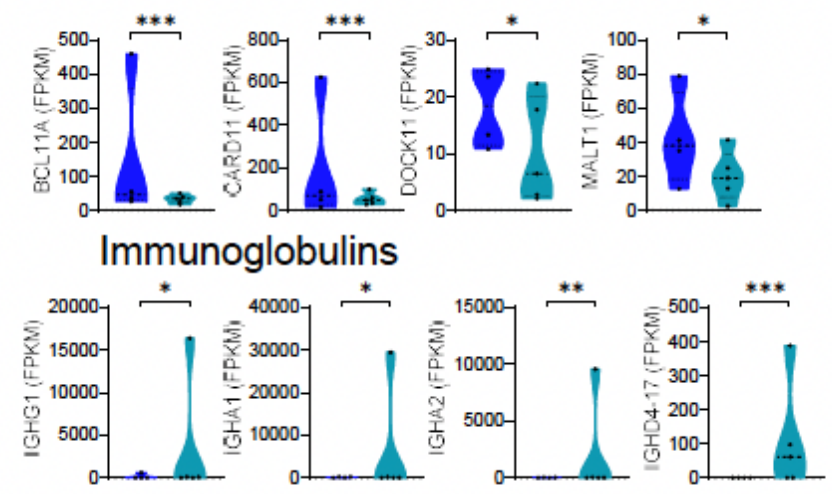
Figure 4

DGEs signatures in $\mathrm{aS}_{1}$-casein-specific $B$ cells from after OIT (desensitized vs tolerance).

(A) PCA plot of 200 DGEs separating allergic groups: before OIT vs after OIT (desensitized and tolerance) vs NT groups. (B) Heatmap of most significant DEGs of aS ${ }_{1}$-casein-specific B cells after OIT; desensitized VS tolerance, $n_{\text {before OIT }}=6$ and $n_{N T}=6$ (C) Violin plots of differentially expressed genes in 8 different gene groups; antigen-binding, BCR signalling, B cell activation, chemotaxis, cytokines/ cytokine receptors, B cell

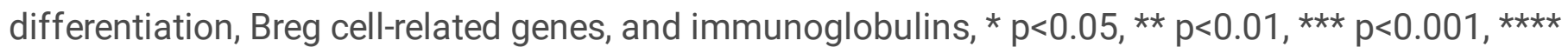
$p<0.0001$. 


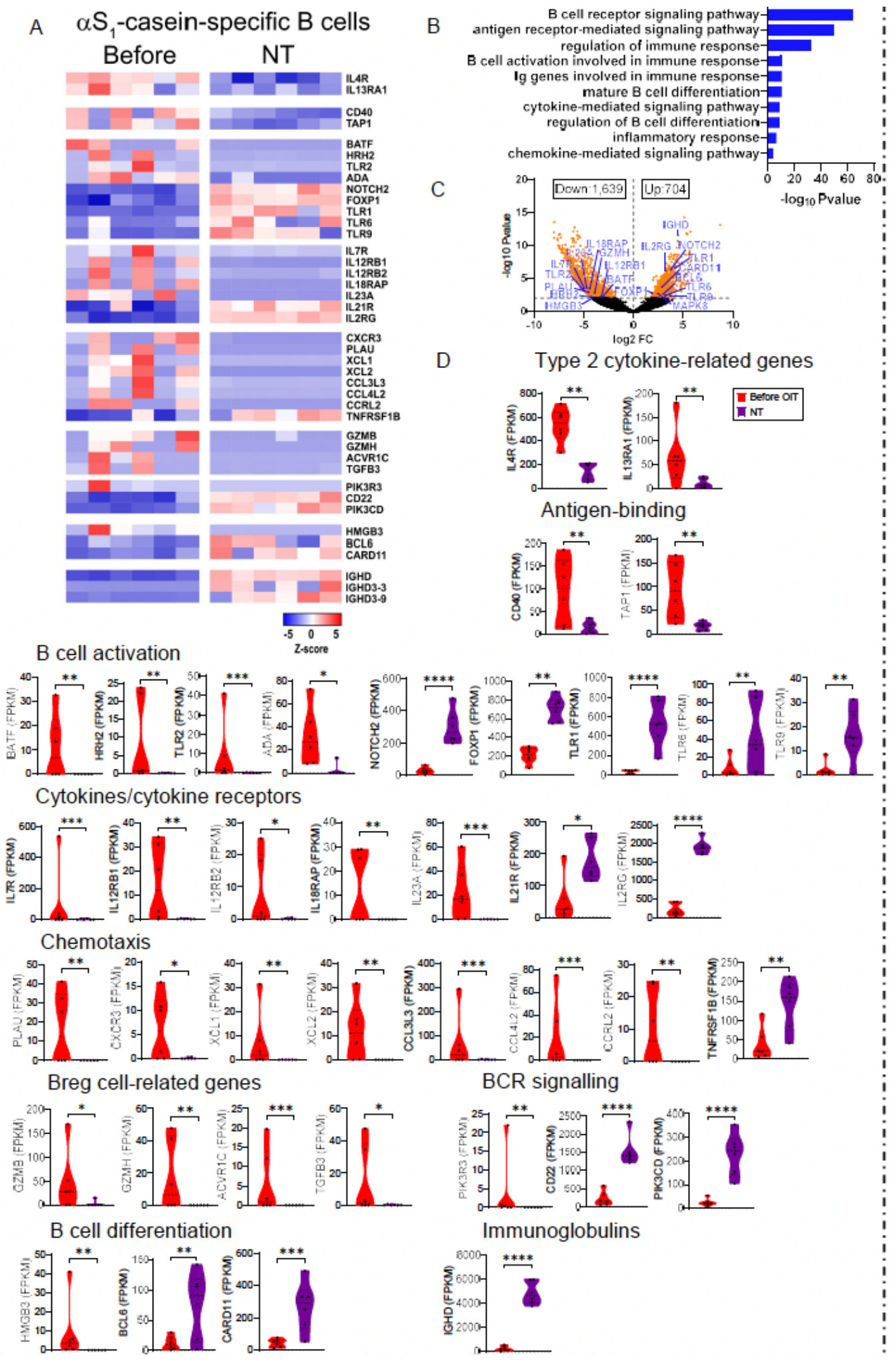

\section{Figure 5}

\section{DGEs signatures in a $\mathrm{S}_{1}$-casein-specific $B$ cells from before OIT vs NT.}

(A) Heatmap showing top significant DEGs of aS $_{1}$-casein-specific $B$ cells from before OIT and NT, $n_{\text {before }}$ OIT $=6$ and $n_{N T}=6$. (B) Top B cell-related pathways analysis (C) Volcano plot shows differentially expressed genes with top 20 significant genes ( $p$-value $<0.05$ ). (D) Violin plots of differentially expressed 
genes in 9 different gene groups; Type 2 cytokine-related genes, antigen-binding, B cell activation, cytokines/ cytokine receptors, chemotaxis, Breg cell-related genes, BCR signalling, B cell differentiation,

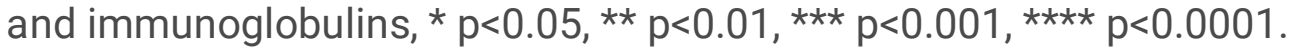

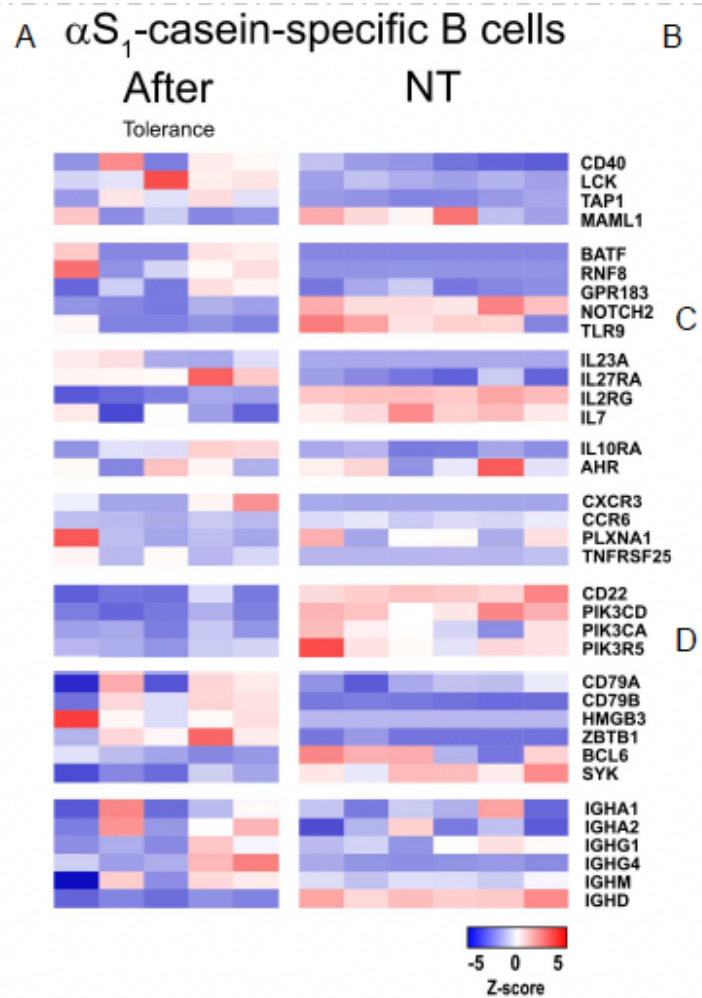

E B cell activation

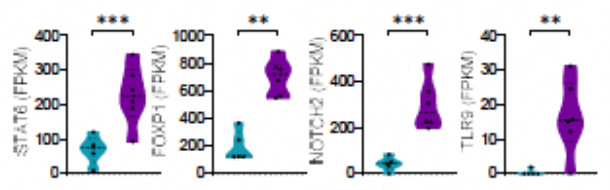

Cytokines/cytokine receptors

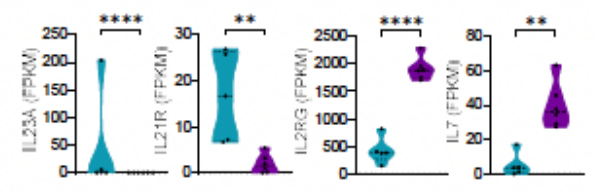

B cell differentiation

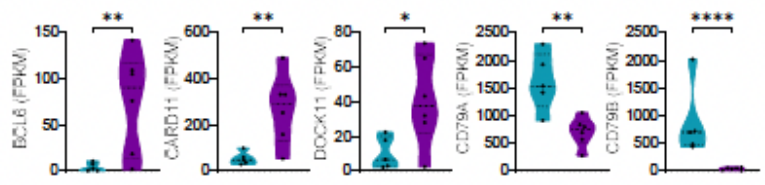

Immunoglobulins
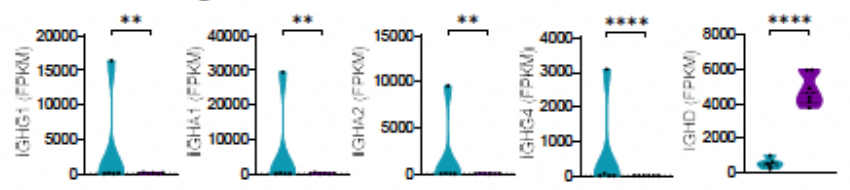

Figure 6
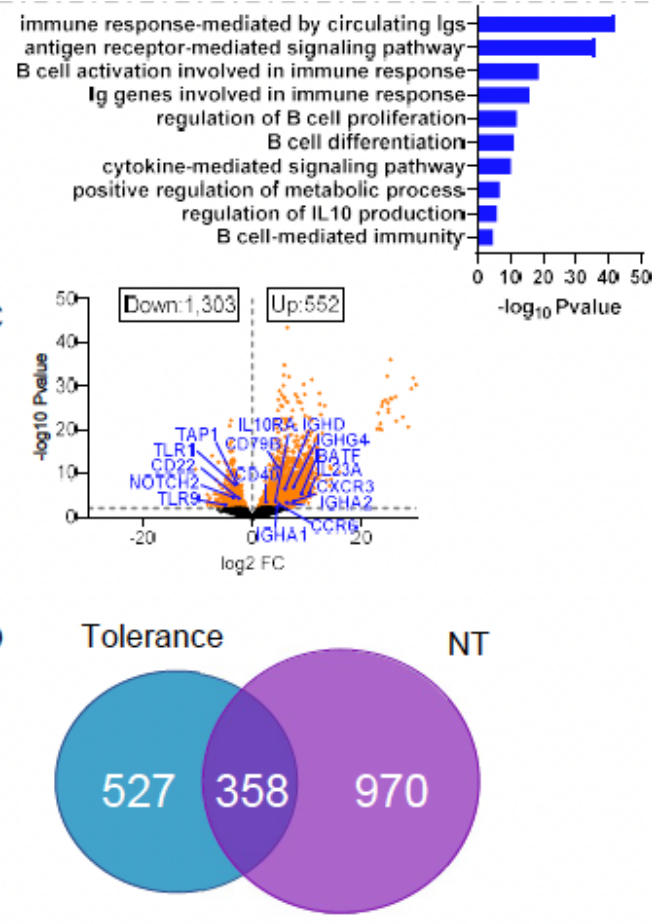

Antigen-binding

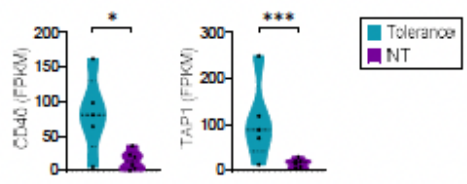

Breg cell-related genes

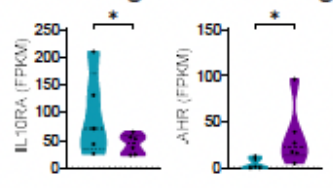

BCR signalling

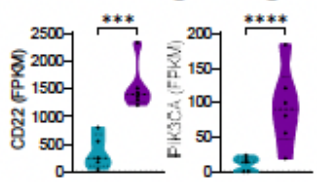




\section{DGEs signatures in a $\mathrm{S}_{1}$-casein-specific $B$ cells from after OIT (tolerance) vs NT.}

(A) Heatmap of the top significant DEGs of aS1-casein-specific B cells from after OIT (tolerance) vs NT, $\mathrm{n}_{\text {after }}$ OIT (tolerance) $=5, \mathrm{n}_{\mathrm{NT}}$ OIT $=6$, (B) Top B cell-related pathways analysis (C) Volcano plot shows differentially expressed genes with top 20 significant genes ( $p$-value $<0.05$ ). (D) Venn-diagram of differentially expressed genes from tolerance vs NT groups ( $p$-value $<0.05$ ). Violin plots of differentially expressed genes in 7 different gene groups; B cell activation, antigen-binding, cytokines/ cytokine receptors, Breg cell-related genes, $B$ cell differentiation, BCR signalling, and immunoglobulins; $(E)$ tolerance vs NT, ${ }^{*} p<0.05, * \star p<0.01, * \star * p<0.001, * \star \star * p<0.0001$. 

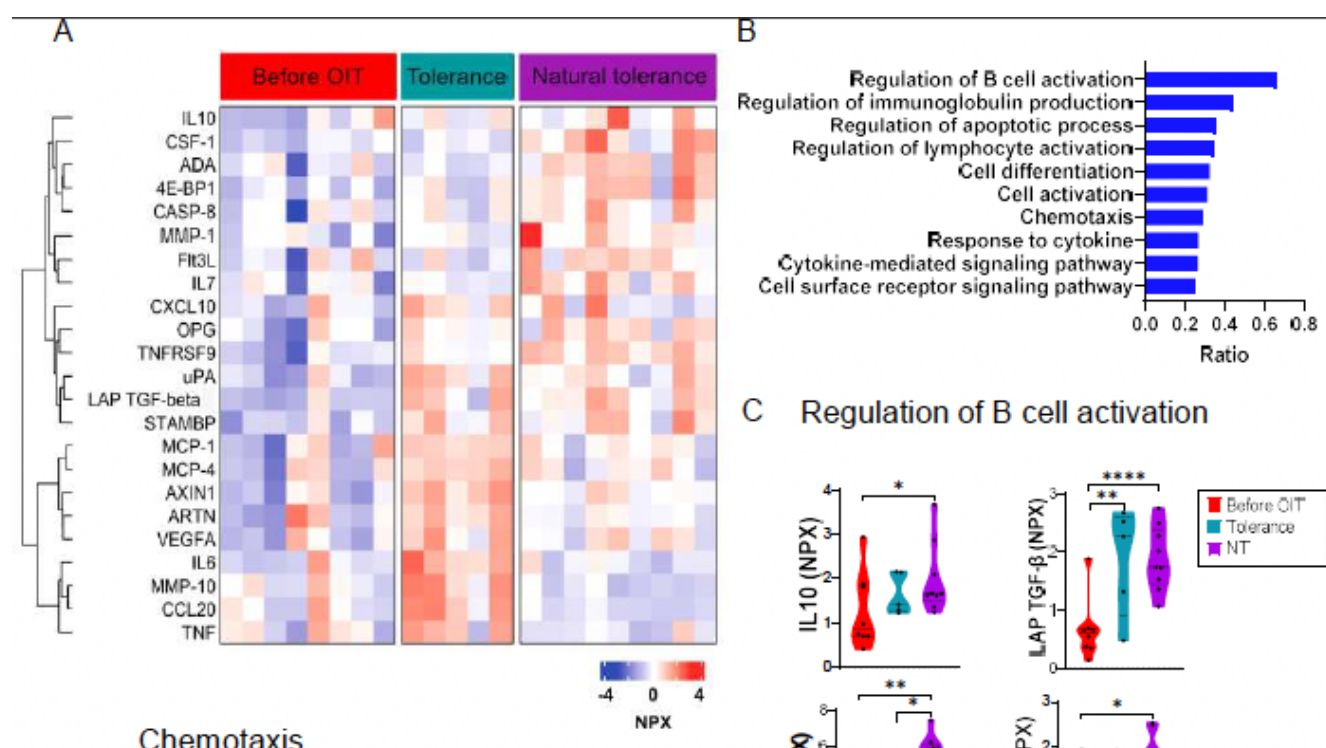

C Regulation of B cell activation
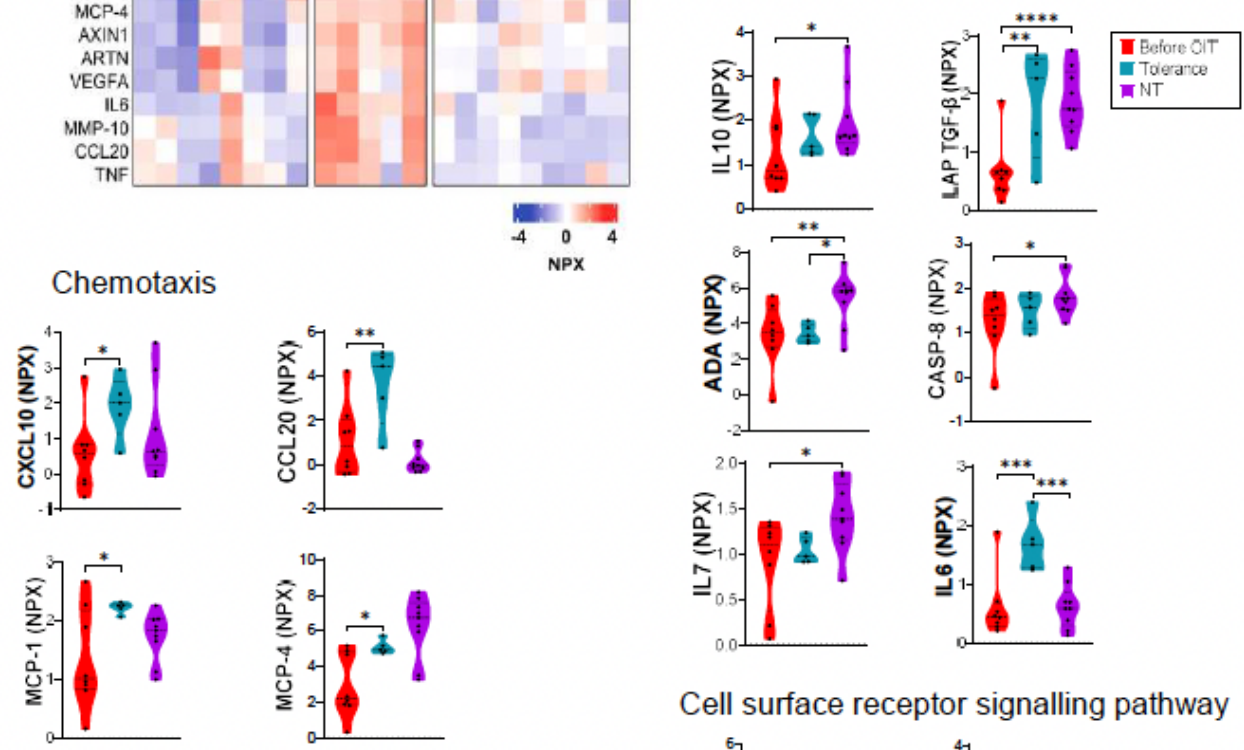

Cell surface receptor signalling pathway
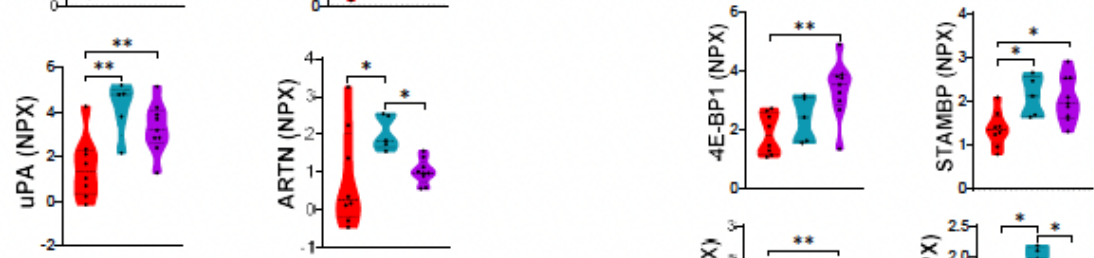

Cytokine-mediated signalling pathway
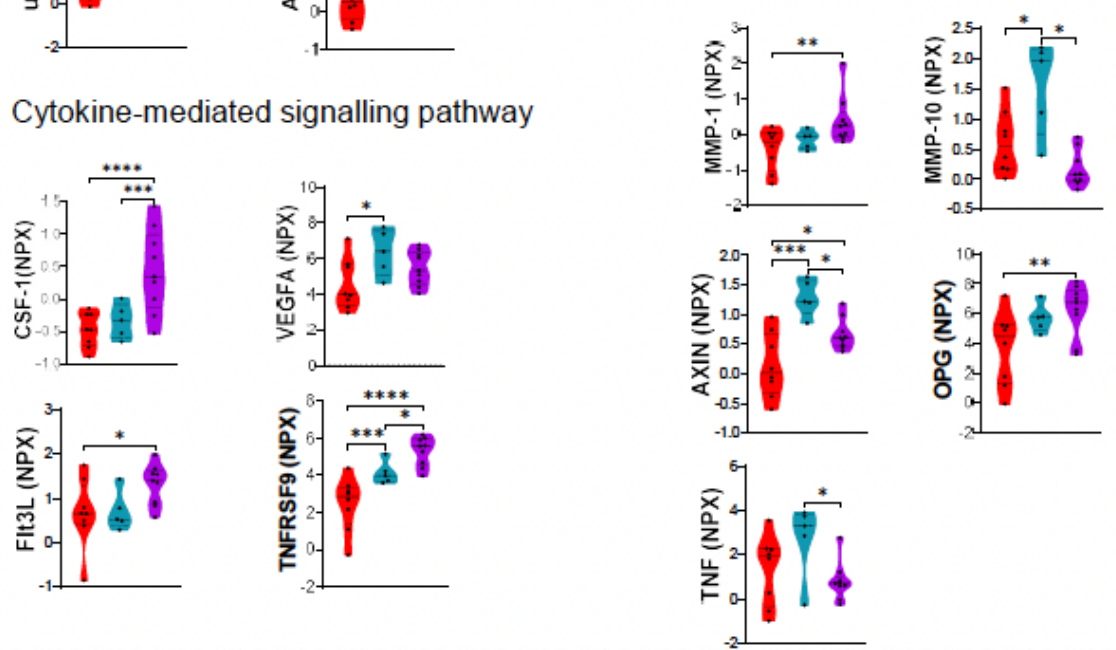

Figure 7

Protein expressions in aS $_{1}$-casein-specific B cell cultures from before OIT, after OIT (desensitized \& tolerance), and NT using proximity extension assay (OLINK).

(A) Heatmap depicts significant differentially secreted proteins from aS ${ }_{1}$-casein-specific $B$ cell supernatants before OIT vs after OIT vs NT. (B) Ratio of 23 significant proteins, for different GO Biological 
processes, to all measured in the Olink inflammation panel. (C) Violin plots of differentially secreted proteins were showed: IL10, TGF- $\beta$, TNFRSF9, AXIN, uPA, STAMBP, OPG, MMP-1, IL7, FIT3L, CASP-8, 4EBP1, ADA, CXCL10, CCL20, VEGF-A, MCP-1, MCP-4, TNF, MMP-10, ARTN, CSF-1, and IL6, * $p<0.05$, ** $p<0.01, * \star * p<0.001, * \star \star * x<0.0001$.

\section{Supplementary Files}

This is a list of supplementary files associated with this preprint. Click to download.

- AMAlphaS1caseinsupplementary.docx

- Supplementaryfigures.pdf

- Table1.docx 